\title{
Backward stochastic differential equations in infinite dimensions with continuous driver and applications
}

\author{
Marco Fuhrman \\ Dipartimento di Matematica, Politecnico di Milano \\ piazza Leonardo da Vinci 32, 20133 Milano, Italy \\ Email: marco.fuhrman@polimi.it \\ Ying $\mathrm{Hu}$ \\ IRMAR, Université Rennes 1 \\ Campus de Beaulieu, 35042 Rennes Cedex, France \\ Email: ying.hu@univ-rennes1.fr
}

Mathematics Subject Classification (2000): 60H10, 60H30.

Keywords. Backward stochastic differential equations, stochastic differential games.

\begin{abstract}
In this paper we prove the existence of solution to backward stochastic differential equations (BSDEs) in infinite dimensions with continuous driver under various assumptions. We apply our results to a stochastic game problem with infinitely many players.
\end{abstract}

\section{Introduction}

In this paper we consider the following backward stochastic differential equation (BSDE), in the sense of [18], on a finite time interval $[0, T]$, in an infinite dimensional setting:

$$
d Y_{t}=-B Y_{t} d t-\psi\left(t, X_{t}, Y_{t}, Z_{t}\right) d t+Z_{t} d W_{t}, \quad Y_{T}=\phi\left(X_{T}\right) .
$$

In the above, $W$ is a cylindrical Wiener process in a Hilbert space $\Xi, B$ is the infinitesimal generator of a strongly continuous dissipative compact semigroup $\left(e^{t B}\right)$ in a Hilbert space $K$, $X$ is a Markov process with respect to the filtration generated by $W, \psi$ and $\phi$ are deterministic functions with values in $K$. The solution $(Y, Z)$ takes values in $K \times L_{2}(\Xi, K)$, where $L_{2}(\Xi, K)$ denotes the space of Hilbert-Schmidt operators from $\Xi$ to $K$. The solution is understood in an appropriate sense, see below.

BSDEs in infinite dimensions were first studied in [17]. In this paper the authors proved existence and uniqueness of the solution to BSDE (1.1) assuming that the driver $\psi$ is uniformly Lipschitz with respect to $(y, z)$.

BSDEs in infinite dimensions were also studied in [1], [2], [3], [10], [14], [19], [21], in the more general case when the driver $\psi$ can be random. In [9], [10], [11], [12], equation (1.1) was considered when the process $X$ takes values in a Hilbert space $H$ and is defined as the solution to a stochastic evolution equation of the form

$$
d X_{t}=A X_{t} d t+F\left(t, X_{t}\right) d t+G\left(t, X_{t}\right) d W_{t}, \quad X_{0}=x \in H .
$$


Here $A$ is the infinitesimal generator of a strongly continuous semigroup $\left(e^{t A}\right)$ in $H, F$ and $G$ are appropriate functions with values in $H$ and in the space of bounded linear operators from $\Xi$ to $H$, respectively. Various problems were considered in these papers, including applications to nonlinear partial differential equations for functions defined on $[0, T] \times H$ and optimal stochastic control. In [13] the fully coupled case is addressed, i.e. when $F$ and $G$ may depend on the unknown processes $Y$ and $Z$.

In this paper we prove existence of a solution to BSDE (1.1) assuming that $\psi$ is only continuous with respect to $(y, z)$.

Our starting point is the result in [15], where all the processes $W, X, Y, Z$ take values in finite-dimensional vector spaces. In that paper $\psi$ is assumed to have linear growth with respect to $(y, z)$; this allows to prove the existence result for the BSDE and to prove existence of a Nash equilibrium in an $N$-player stochastic differential game. A crucial assumption in that paper is a condition on the densities of transition probabilities of the process $X$ with respect to the Lebesgue measure. This condition is fulfilled in the case when $G$ is uniformly non degenerate. The result of [15] was generalized in [16] to the case of discrete-functional-type drivers.

In our paper we also impose conditions on the transition probabilities of the process $X$. However, due to the infinite dimensional nature of the state space $H$, we need completely different assumptions.

In section 3 we consider the case when $X$ is an Ornstein-Uhlenbeck process, i.e. it solves (1.2) with $F=0$ and $G$ constant. In this case explicit conditions are known to ensure equivalence of transition probabilities. We prove a formula for mutual densities, generalizing a result in [4], and use it to prove the existence of a solution to (1.1) assuming that $\psi$ has linear growth with respect to $(y, z)$. Generalizations of this result to more general processes $X$ seem to be possible, for instance using the formulae for transition densities introduced in [22], [23], [24]. The present result is however sufficient for the applications to stochastic games that we present.

In section 6 we apply the existence result for the BSDE to prove existence of a Nash equilibrium in a stochastic game. The underlying controlled process has a nonlinear drift and constant diffusion coefficient: see equation (6.1). This time, using the infinite-dimensionality of the process $Y$, we are able to study a stochastic game with infinitely many players. Stochastic games with an infinite number of players are a mathematical model used to describe a variety of economical and financial markets, but so far a dynamical setting with continuous time was not considered to our knowledge, perhaps due to the complexity of the techniques involved.

In sections 4 and 5 we only assume that $X$ is a Markov process with values in a metric space, and we prove the existence of solution to the BSDE assuming that $\psi$ is bounded. We impose two kinds of conditions. First, in section 4, we require the transition probabilities of $X$ to be equivalent to each other (but no condition is imposed on the corresponding densities). An application is given in example 4.1, again in the case of a process solution of an evolution equation of the form (1.2). In section 5 we address a case where transition probabilities can be even singular, and we require a continuity condition with respect to the variation norm: see (5.2). This kind of property is customary in the theory of stochastic evolution equations in infinite-dimensional spaces: it has been deeply investigated in connection with the so-called strong Feller property and several conditions are known which guarantee that it is verified: see [7]. One example is given below, see example 5.1, to show applicability of the general result.

In section 2 we introduce notation, we state a general approximation lemma and recall some facts about the Ornstein-Uhlenbeck process in a Hilbert space.

Some results of this paper have been announced at the Fourth International Conference on Backward Stochastic Differential Equations and Applications, Shanghai, May 30th - June 1st, 2005. The first author would like to thank the Université de Rennes 1 for his stay during which this article was begun. 


\section{Preliminaries}

In this section we collect material that will be used in the sequel. First we recall some notation, then we define the Ornstein-Uhlenbeck semigroup that is used in sections 3 and 6, finally we state and prove an approximation lemma that is frequently used afterwards.

\section{$2.1 \quad$ Notation}

In this paper the letters $H, K, \Xi$ denote Hilbert spaces. All Hilbert spaces are assumed to be real and separable. The norm is denoted $|\cdot|$ and the scalar product $\langle\cdot, \cdot\rangle$, with a subscript to indicate the space, if necessary. $L(H, K)$ denotes the space of linear bounded operators from $H$ to $K$, with its usual norm. We shorten $L(H, H)$ to $L(H) . L_{2}(H, K)$ denotes the space of Hilbert-Schmidt operators from $H$ to $K$, with the Hilbert-Schmidt norm. Operator norms are also denoted by $|\cdot|$, with a subscript if necessary.

Let $(\Omega, \mathcal{F}, \mathbb{P})$ be a complete probability space. A cylindrical Wiener process $\left\{W_{t}, t \geq 0\right\}$ in a Hilbert space $\Xi$ is a family of linear mappings $\xi \rightarrow W_{t}^{\xi}$, defined for $\xi \in \Xi$ with values in $L^{2}(\Omega, \mathcal{F}, \mathbb{P})$, such that $\left\{W_{t}^{\xi}, t \geq 0\right\}$ is a real Wiener process and $\mathbb{E}\left[W_{t}^{\xi} W_{s}^{\eta}\right]=(t \wedge s)\langle\xi, \eta\rangle$ for $\xi, \eta \in \Xi$ and $t, s \geq 0$. By $\mathcal{F}_{t}$ we denote the $\sigma$-algebra generated by the random variables $\left\{W_{s}^{\xi}, s \in[0, t], \xi \in \Xi\right\}$ and by the $\mathbb{P}$-null sets of $\mathcal{F}$. We call $\left(\mathcal{F}_{t}\right)_{t \geq 0}$ the Brownian filtration of $W$.

Stochastic integration theory can be defined with respect to $W$ : we refer to [6] for details. If $\left\{\Psi_{t}, t \in[0, T]\right\}$ is an $\left(\mathcal{F}_{t}\right)$-predictable process with values in $L_{2}(\Xi, H)$, satisfying

P-a.s. $\int_{0}^{T}\left|\Psi_{t}\right|_{L_{2}(\Xi, H)}^{2}<\infty$ then the stochastic integral $\left\{\int_{0}^{t} \Psi_{s} d W_{s}, t \in[0, T]\right\}$ is an $\left(\mathcal{F}_{t}\right)$-local martingale with values in $H$ admitting a continuous version.

\subsection{The Ornstein-Uhlenbeck process}

Let $H, \Xi$ be Hilbert spaces. We are given two linear operators $A: D(A) \subset H \rightarrow H$ and $G \in L(\Xi, H)$ such that

Hypothesis 1 (i) The operator $A: D(A) \subset H \rightarrow H$ is the infinitesimal generator of a strongly continuous semigroup $\left\{e^{t A}, t \geq 0\right\}$ of bounded linear operators in $H$.

(ii) $G: \Xi \rightarrow H$ is a bounded linear operator.

(iii) The operators

$$
Q_{t} x=\int_{0}^{t} e^{s A} G G^{*} e^{s A^{*}} x d s, x \in H
$$

are of trace class for all $t \geq 0$.

(iv) $e^{t A}(H) \subset Q_{t}^{\frac{1}{2}}(H)$, for all $t>0$.

We define the Ornstein-Uhlenbeck process as the solution of the following stochastic equation:

$$
d X_{t}=A X_{t} d t+G d W_{t}, \quad X_{0}=x
$$

where $x \in H$ is given and $W$ is a cylindrical Wiener process in $\Xi$. Equation (2.1) is understood in the so-called mild sense: the solution is by definition the process

$$
X_{t}=e^{t A} x+\int_{0}^{t} e^{(t-s) A} G d W_{s}, \quad t \geq 0 .
$$

It is well known (see e.g. [6]) that under the assumptions $(i)-($ iii $)$ in Hypothesis 1 the Ito integral is well defined and $X_{t}$ is a random variable with values in $H$ with law $\mathcal{N}\left(e^{t A} x, Q_{t}\right)$, i.e. 
the Gaussian measure with mean $e^{t A} x$ and covariance operator $Q_{t}$. Moreover, condition (iv) ensures that the measures $\left\{\mathcal{N}\left(e^{t A} x, Q_{t}\right), t>0, x \in H\right\}$ are all equivalent. In the following we fix $0<t \leq T, x \in H$ and we denote by $k_{t T}(x, \cdot)$ the density of $\mathcal{N}\left(e^{t A} x, Q_{t}\right)$ with respect to $\mathcal{N}\left(0, Q_{T}\right)$.

Lemma 2 Assume that Hypothesis 1 holds, and let $0<t \leq T$ and $x \in H$ be given. Define

$$
\Theta_{t T}=Q_{T}^{-\frac{1}{2}} e^{t A} Q_{T-t}\left(Q_{T}^{-\frac{1}{2}} e^{t A}\right)^{*} .
$$

Then $1-\Theta_{t T}$ is a positive operator with bounded inverse and we have, for $\mathcal{N}\left(0, Q_{T}\right)$-almost every $y \in H$,

$$
\begin{aligned}
k_{t T}(x, y)= & \operatorname{det}\left(1-\Theta_{t T}\right)^{-\frac{1}{2}} \exp \left\{-\frac{1}{2}\left\langle\left(1-\Theta_{t T}\right)^{-1} Q_{T}^{-\frac{1}{2}} e^{t A} x, Q_{T}^{-\frac{1}{2}} e^{t A} x\right\rangle\right. \\
& \left.+\left\langle\left(1-\Theta_{t T}\right)^{-1} Q_{T}^{-\frac{1}{2}} e^{t A} x, Q_{T}^{-\frac{1}{2}} y\right\rangle-\frac{1}{2}\left\langle\Theta_{t T}\left(1-\Theta_{t T}\right)^{-1} Q_{T}^{-\frac{1}{2}} y, Q_{T}^{-\frac{1}{2}} y\right\rangle\right\}
\end{aligned}
$$

We also have the following estimates:

$$
\left|\left(1-\Theta_{t T}\right)^{-1}\right| \leq 1+\left|Q_{T-t}\right|\left|Q_{t}^{-1 / 2} e^{t A}\right|^{2}
$$

and

$$
\operatorname{det}\left(1-\Theta_{t T}\right)^{-1} \leq \exp \left\{\left(1+\left|Q_{T-t}\right|\left|Q_{t}^{-1 / 2} e^{t A}\right|^{2}\right)\left|Q_{t}^{-1 / 2} e^{t A}\right|^{2} \text { Trace } Q_{T-t}\right\} .
$$

By 1 we also denote the identity operator. These formulae need some explanations. First we note that, as a consequence of Hypothesis 1 , one can prove that the operators $Q_{T}^{-1 / 2} e^{t A}$ and $Q_{t}^{-1 / 2} e^{t A}$ are everywhere defined and bounded and that $\Theta_{t T}$ is a symmetric trace class operator satisfying $0 \leq \Theta_{t T}<1$. Next, the determinant occurring in (2.4) and (2.6) is understood as the infinite product of eigenvalues. It is well defined, since $\Theta_{t T}$ is trace class. Finally, for arbitrary $b \in H$ and trace class symmetric operator $M$ the functions $\left\langle b, Q_{T}^{-1 / 2} y\right\rangle$ and $\left\langle M Q_{T}^{-1 / 2} y, Q_{T}^{-1 / 2} y\right\rangle$, $y \in H$, are defined by the formulae

$$
\left\langle b, Q_{T}^{-1 / 2} y\right\rangle=\sum_{j=1}^{\infty} \lambda_{j}^{-1 / 2}\left\langle b, e_{j}\right\rangle\left\langle y, e_{j}\right\rangle,
$$

and

$$
\left\langle M Q_{T}^{-1 / 2} y, Q_{T}^{-1 / 2} y\right\rangle=\sum_{j, k=1}^{\infty} \lambda_{j}^{-1 / 2} \lambda_{k}^{-1 / 2}\left\langle M e_{k}, e_{j}\right\rangle\left\langle y, e_{j}\right\rangle\left\langle y, e_{k}\right\rangle,
$$

where $\left(e_{k}\right),\left(\lambda_{k}\right)$ are the eigenvectors and eigenvalues of $Q_{T}$, the eigenvalues are strictly positive. The series converge in $L^{2}\left(H, \mathcal{N}\left(0, Q_{T}\right)\right)$ so that the formula $(2.4)$ defines a function $k_{t T}(x, \cdot)$ up to a set of $\mathcal{N}\left(0, Q_{T}\right)$ measure 0 . In particular, the function $y \rightarrow\left\langle b, Q_{T}^{-1 / 2} y\right\rangle$ defined in (2.7) has centered gaussian law with covariance $|b|^{2}$ on the probability space $\left(H, \mathcal{N}\left(0, Q_{T}\right)\right)$ and it follows that

$$
\int_{H} \exp \left\{\left\langle b, Q_{T}^{-1 / 2} y\right\rangle\right\} \mathcal{N}\left(0, Q_{T}\right)(d y)=\exp \left\{\frac{1}{2}|b|^{2}\right\} .
$$

Lemma 2 is similar to Proposition 4.2 in [4], where densities with respect to invariant measure of the process $X$ were considered instead of densities with respect to $\mathcal{N}\left(0, Q_{T}\right)$. Here we do not assume that $X$ has an invariant measure. The proof of Lemma 2 is postponed to the appendix. 


\subsection{An approximation procedure}

Lemma 3 Let $M$ be a metric space, $H$ and $K$ Hilbert spaces and $\psi: M \times H \rightarrow K$ a Borel measurable function satisfying

$$
|\psi(m, h)| \leq C(|h|+g(m)), \quad m \in M, h \in H
$$

for some constant $C>0$ and some function $g: M \rightarrow[0, \infty)$. Let $\psi(m, \cdot): H \rightarrow K$ be $a$ continuous function for every $m \in M$.

Then there exists a sequence of Borel measurable functions $\psi_{n}: M \times H \rightarrow K$ satisfying the following conditions.

(i) There exists a constant $C^{\prime}>0$ such that for every $n$

$$
\left|\psi_{n}(m, h)\right| \leq C^{\prime}(|h|+g(m)+1), \quad m \in M, h \in H .
$$

(ii) For every $m \in M, \psi_{n}(m, \cdot): H \rightarrow K$ is infinitely Fréchet differentiable.

(iii) There exist constants $C_{n}>0$ such that for every $n$

$$
\left|\psi_{n}(m, h)-\psi_{n}(m, k)\right| \leq C_{n}|h-k|, \quad m \in M ; h, k \in H .
$$

(iv) If $h_{n} \rightarrow h$ in $H$ then $\psi_{n}\left(m, h_{n}\right) \rightarrow \psi(m, h)$ in $K$, for every $m \in M$.

Proof. We use the construction in [20]. Let $\left(e_{i}\right)$ denote a basis of $H$ and define the projection $P_{n}: H \rightarrow \mathbb{R}^{n}$ setting $P_{n} h=\left(\left\langle e_{i}, h\right\rangle\right)_{i=1}^{n}, h \in H$. Then for $y=\left(y_{i}\right)_{i=1}^{n} \in \mathbb{R}^{n}$ we have $P_{n}^{*} y=\sum_{i=1}^{n} y_{i} e_{i}$. Let $\rho_{n}: \mathbb{R}^{n} \rightarrow[0, \infty)$ be infinitely differentiable functions such that $\int_{\mathbb{R}^{n}} \rho_{n}(y) d y=1$ with support contained in $\left\{y \in \mathbb{R}^{n}:|y|_{\mathbb{R}^{n}} \leq 1 / n\right\}$. Define

$$
\bar{\psi}_{n}(m, h)=\int_{\mathbb{R}^{n}} \psi\left(m, P_{n}^{*}\left(P_{n} h+y\right)\right) \rho_{n}(y) d y, \quad h \in H, m \in M .
$$

It is easy to show that $\bar{\psi}_{n}(m, \cdot): H \rightarrow K$ is infinitely Fréchet differentiable, that $\bar{\psi}_{n}\left(m, h_{n}\right) \rightarrow$ $\psi(m, h)$ whenever $h_{n} \rightarrow h$ in $H$, and to prove the estimate $\left|\bar{\psi}_{n}(m, h)\right| \leq C^{\prime}(|h|+g(m)+1)$, for some constant $C^{\prime}$. Next we take $\eta_{n} \in C^{\infty}(\mathbb{R})$ such that $\eta_{n}(x)=1$ for $x \leq n, \eta_{n}(x)=0$ for $x \geq n+1,\left|\eta_{n}(x)\right|+\left|\eta_{n}^{\prime}(x)\right| \leq c$ for some constant $c$. Then setting

$$
\psi_{n}(m, h)=\eta_{n}\left(\sqrt{1+|h|^{2}}-1+g(m)\right) \bar{\psi}_{n}(m, h), \quad h \in H, m \in M,
$$

it is easy to show that the gradient of $\psi_{n}$ is bounded by some constant (depending on $n$ ) and that all the conclusions of the Lemma are satisfied.

\section{BSDE with linear growth continuous driver}

In this section we consider a BSDE of the form:

$$
d Y_{t}=-B Y_{t} d t-\psi\left(t, X_{t}, Y_{t}, Z_{t}\right) d t+Z_{t} d W_{t}, \quad Y_{T}=\phi\left(X_{T}\right),
$$

for $t$ varying on a bounded time interval $[0, T]$. $W$ is a cylindrical Wiener process in a Hilbert space $\Xi$ and we denote by $\left(\mathcal{F}_{t}\right)$ its Brownian filtration. The unknown processes $Y$ and $Z$ take values in a Hilbert space $K$ and in the Hilbert space $L_{2}(\Xi, K)$ respectively. $X$ is a given $\left(\mathcal{F}_{t}\right)$ predictable process in another Hilbert space $H$. On the drivers $B$ and $\psi$ and the final datum $\phi$ we assume the following. 
Hypothesis 4 (i) The operator $B: D(B) \subset K \rightarrow K$ is the infinitesimal generator of a strongly continuous dissipative semigroup $\left\{e^{t B}, t \geq 0\right\}$ of linear bounded operators on $K$.

(ii) $\phi: H \rightarrow K$ and $\psi:[0, T] \times H \times K \times L_{2}(\Xi, K) \rightarrow K$ are Borel measurable functions, and there exist two constants $C>0$ and $p \geq 1$ such that

$$
\begin{gathered}
|\phi(x)| \leq C\left(1+|x|^{p}\right), \quad x \in H, \\
|\psi(t, x, y, z)| \leq C\left(1+|x|^{p}+|y|+|z|\right), \quad t \in[0, T], x \in H, y \in K, z \in L_{2}(\Xi, K) .
\end{gathered}
$$

(iii) For every $t \in[0, T]$ and $x \in H$, the function $\psi(t, x, \cdot, \cdot): K \times L_{2}(\Xi, K) \rightarrow K$ is continuous.

Let us suppose that $\sup _{t \in[0, T]} \mathbb{E}\left|X_{t}\right|^{2 p}<\infty$. We say that an $\left(\mathcal{F}_{t}\right)$-predictable process $(Y, Z)$ with values in $K \times L_{2}(\Xi, K)$ is a mild solution of (3.1) if

$$
\sup _{t \in[0, T]} \mathbb{E}\left|Y_{t}\right|^{2}+\mathbb{E} \int_{0}^{T}\left|Z_{t}\right|^{2} d t<\infty
$$

and for every $t \in[0, T]$ the following equality holds:

$$
Y_{t}+\int_{t}^{T} e^{(s-t) B} Z_{s} d W_{s}=e^{(T-t) B} \phi\left(X_{T}\right)+\int_{t}^{T} e^{(s-t) B} \psi\left(s, X_{s}, Y_{s}, Z_{s}\right) d s, \quad \mathbb{P}-\text { a.s. }
$$

The result of [17] states that there exists a unique mild solution if, in addition to the previous assumptions, one supposes that the function $\psi(t, x, \cdot, \cdot)$ is Lipschitz continuous. In the following we will drop the Lipschitz condition and prove some existence results. We first need some preliminary estimates.

Lemma 5 Assume that Hypothesis 4 holds and let $X$ be an $\left(\mathcal{F}_{t}\right)$-predictable process satisfying $\sup _{t \in[0, T]} \mathbb{E}\left|X_{t}\right|^{2 p}<\infty$. Let $(Y, Z)$ be a mild solution to (3.1). Then

$$
\sup _{t \in[0, T]} \mathbb{E}\left|Y_{t}\right|^{2}+\mathbb{E} \int_{0}^{T}\left|Z_{t}\right|^{2} d t \leq C \sup _{t \in[0, T]} \mathbb{E}\left(1+\left|X_{t}\right|^{2 p}\right) .
$$

If $\psi^{\prime}, \phi^{\prime}$ are functions satisfying Hypothesis 4 and $\left(Y^{\prime}, Z^{\prime}\right)$ is a corresponding mild solution then

$\mathbb{E} \int_{0}^{T}\left|Z_{t}-Z_{t}^{\prime}\right|^{2} d t \leq \mathbb{E}\left|\phi\left(X_{T}\right)-\phi^{\prime}\left(X_{T}\right)\right|^{2}+C\left(\sup _{t \in[0, T]} \mathbb{E}\left(1+\left|X_{t}\right|^{2 p}\right)\right)^{1 / 2}\left(\mathbb{E} \int_{0}^{T}\left|Y_{t}-Y_{t}^{\prime}\right|^{2} d t\right)^{1 / 2}$.

In (3.4) and (3.5) the constant $C$ depends only on $T$ and on the constants $C, p$ in Hypothesis 4.

Proof. Let us introduce the operators $J_{k}=k(k \cdot 1-B)^{-1}, k>0$. A direct computation shows that $B J_{k}=k^{2}(k \cdot 1-B)^{-1}-k \cdot 1$, so in particular the operators $B J_{k}$ are bounded (they are called the Yosida approximations of $B)$. We set $Y_{t}^{k}=J_{k} Y_{t}, Z_{t}^{k}=J_{k} Z_{t}$. We now verify that $Y^{k}$ admits the Itô differential

$$
d Y_{t}^{k}=-B Y_{t}^{k} d t-J_{k} \psi\left(t, X_{t}, Y_{t}, Z_{t}\right) d t+Z_{t}^{k} d W_{t} .
$$

In fact applying $J_{k}$ to both sides of (3.3) we have

$$
Y_{t}^{k}+\int_{t}^{T} e^{(s-t) B} Z_{s}^{k} d W_{s}=e^{(T-t) B} J_{k} \phi\left(X_{T}\right)+\int_{t}^{T} e^{(s-t) B} J_{k} \psi\left(s, X_{s}, Y_{s}, Z_{s}\right) d s .
$$


Applying $B$ to both sides and integrating we obtain, for every $r \in[0, T]$,

$$
\begin{array}{rl}
\int_{r}^{T} & B Y_{t}^{k} d t+\int_{r}^{T} \int_{t}^{T} e^{(s-t) B} B Z_{s}^{k} d W_{s} d t \\
& =\int_{r}^{T} e^{(T-t) B} B J_{k} \phi\left(X_{T}\right) d t+\int_{r}^{T} \int_{t}^{T} e^{(s-t) B} B J_{k} \psi\left(s, X_{s}, Y_{s}, Z_{s}\right) d s d t .
\end{array}
$$

We have

$$
\int_{r}^{T} e^{(T-t) B} B J_{k} \phi\left(X_{T}\right) d t=e^{(T-r) B} J_{k} \phi\left(X_{T}\right)-J_{k} \phi\left(X_{T}\right)
$$

and, applying the stochastic Fubini theorem (see e.g. [6])

$$
\int_{r}^{T} \int_{t}^{T} e^{(s-t) B} B Z_{s}^{k} d W_{s} d t=\int_{r}^{T} \int_{r}^{s} e^{(s-t) B} B Z_{s}^{k} d t d W_{s}=\int_{r}^{T}\left(e^{(s-r) B} Z_{s}^{k}-Z_{s}^{k}\right) d W_{s} .
$$

Substituting in (3.8) and comparing with (3.7) gives

$$
\int_{r}^{T} B Y_{t}^{k} d t=Y_{r}^{k}+\int_{r}^{T} Z_{s}^{k} d W_{s}-J_{k} \phi\left(X_{T}\right)-\int_{r}^{T} J_{k} \psi\left(s, X_{s}, Y_{s}, Z_{s}\right) d s,
$$

which proves (3.6).

Applying the Itô formula to $\left|Y_{t}^{k}\right|^{2}$ we obtain

$$
\begin{aligned}
& \left|Y_{t}^{k}\right|^{2}+\int_{t}^{T}\left|Z_{s}^{k}\right|^{2} d s \\
& =\left|J_{k} \phi\left(X_{T}\right)\right|^{2}+2 \int_{t}^{T}\left(\left\langle Y_{s}^{k}, B Y_{s}^{k}\right\rangle+\left\langle Y_{s}^{k}, J_{k} \psi\left(s, X_{s}, Y_{s}, Z_{s}\right)\right\rangle\right) d s-2 \int_{t}^{T}\left\langle Y_{s}^{k}, Z_{s}^{k} d W_{s}\right\rangle .
\end{aligned}
$$

We have

$$
\mathbb{E}\left(\int_{0}^{T}\left|\left(Z_{s}^{k}\right)^{*} Y_{s}^{k}\right|^{2} d s\right)^{1 / 2} \leq \mathbb{E}\left[\sup _{s \in[0, T]}\left|Y_{s}^{k}\right|\left(\int_{0}^{T}\left|Z_{s}^{k}\right|^{2} d s\right)^{1 / 2}\right]<\infty,
$$

since it follows from (3.6) and Burkholder's inequality that $\mathbb{E} \sup _{t \in[0, T]}\left|Y_{t}^{k}\right|^{2}<\infty$. (3.9) ensures that we can take expectation in the previous equality and obtain

$$
\mathbb{E}\left|Y_{t}^{k}\right|^{2}+\mathbb{E} \int_{t}^{T}\left|Z_{s}^{k}\right|^{2} d s=\mathbb{E}\left|J_{k} \phi\left(X_{T}\right)\right|^{2}+2 \mathbb{E} \int_{t}^{T}\left(\left\langle Y_{s}^{k}, B Y_{s}^{k}\right\rangle+\left\langle Y_{s}^{k}, J_{k} \psi\left(s, X_{s}, Y_{s}, Z_{s}\right)\right\rangle\right) d s .
$$

Now we use the dissipativity of $B$ and we obtain

$$
\mathbb{E}\left|Y_{t}^{k}\right|^{2}+\mathbb{E} \int_{t}^{T}\left|Z_{s}^{k}\right|^{2} d s \leq \mathbb{E}\left|J_{k} \phi\left(X_{T}\right)\right|^{2}+2 \mathbb{E} \int_{t}^{T}\left\langle Y_{s}^{k}, J_{k} \psi\left(s, X_{s}, Y_{s}, Z_{s}\right)\right\rangle d s .
$$

It is well known that $\left|J_{k}\right|_{L(K)} \leq 1$ and $J_{k} h \rightarrow h$ for every $h \in K$. By the growth condition on $\psi$, the hypothesis $\sup _{t \in[0, T]} \mathbb{E}\left|X_{t}\right|^{2 p}<\infty$ and by (3.2) we can apply the dominated convergence theorem and we arrive at

$$
\mathbb{E}\left|Y_{t}\right|^{2}+\mathbb{E} \int_{t}^{T}\left|Z_{s}\right|^{2} d s \leq \mathbb{E}\left|\phi\left(X_{T}\right)\right|^{2}+2 \mathbb{E} \int_{t}^{T}\left\langle Y_{s}, \psi\left(s, X_{s}, Y_{s}, Z_{s}\right)\right\rangle d s .
$$

Next we have, for every $\epsilon>0$ and for some constant $C_{\epsilon}$,

$$
\left\langle Y_{s}, \psi\left(s, X_{s}, Y_{s}, Z_{s}\right)\right\rangle \leq C\left|Y_{s}\right|\left(1+\left|X_{s}\right|^{p}+\left|Y_{s}\right|+\left|Z_{s}\right|\right) \leq \epsilon\left|Z_{s}\right|^{2}+C_{\epsilon}\left(1+\left|X_{s}\right|^{2 p}+\left|Y_{s}\right|^{2}\right) .
$$


Choosing $\epsilon$ sufficiently small we obtain, for some $C, c>0$,

$$
\begin{aligned}
\mathbb{E}\left|Y_{t}\right|^{2}+c \mathbb{E} \int_{t}^{T}\left|Z_{s}\right|^{2} d s & \leq \mathbb{E}\left|\phi\left(X_{T}\right)\right|^{2}+2 \mathbb{E} \int_{t}^{T}\left(1+\left|X_{s}\right|^{2 p}+\left|Y_{s}\right|^{2}\right) d s \\
& \leq C \sup _{t \in[0, T]}\left(1+\mathbb{E}\left|X_{t}\right|^{2 p}\right)+C \mathbb{E} \int_{t}^{T}\left|Y_{s}\right|^{2} d s
\end{aligned}
$$

and (3.4) follows from Gronwall's lemma.

In order to prove (3.5) we write the equation satisfied by $\left(Y-Y^{\prime}, Z-Z^{\prime}\right)$ and, introducing the operators $J_{k}$ and proceeding as before, instead of (3.10) we arrive at

$$
\begin{aligned}
\mathbb{E} \mid Y_{t} & -\left.Y_{t}^{\prime}\right|^{2}+\mathbb{E} \int_{t}^{T}\left|Z_{s}-Z_{s}^{\prime}\right|^{2} d s \\
& \leq \mathbb{E}\left|\phi\left(X_{T}\right)-\phi^{\prime}\left(X_{T}\right)\right|^{2}+2 \mathbb{E} \int_{t}^{T}\left\langle Y_{s}-Y_{s}^{\prime}, \psi\left(s, X_{s}, Y_{s}, Z_{s}\right)-\psi^{\prime}\left(s, X_{s}, Y_{s}^{\prime}, Z_{s}^{\prime}\right)\right\rangle d s .
\end{aligned}
$$

We set $f_{s}=\psi\left(s, X_{s}, Y_{s}, Z_{s}\right)-\psi^{\prime}\left(s, X_{s}, Y_{s}^{\prime}, Z_{s}^{\prime}\right)$ and note that

$$
\left|f_{s}\right| \leq C\left(1+\left|X_{s}\right|^{p}+\left|Y_{s}\right|+\left|Z_{s}\right|+\left|Y_{s}^{\prime}\right|+\left|Z_{s}^{\prime}\right|\right) .
$$

From estimate (3.4) we deduce

$$
\mathbb{E} \int_{0}^{T}\left|f_{s}\right|^{2} d s \leq C \sup _{t \in[0, T]} \mathbb{E}\left(1+\left|X_{t}\right|^{2 p}\right)
$$

and we obtain

$$
\begin{aligned}
\mathbb{E} \mid Y_{t} & -\left.Y_{t}^{\prime}\right|^{2}+\mathbb{E} \int_{t}^{T}\left|Z_{s}-Z_{s}^{\prime}\right|^{2} d s \\
& \leq \mathbb{E}\left|\phi\left(X_{T}\right)-\phi^{\prime}\left(X_{T}\right)\right|^{2}+2\left(\mathbb{E} \int_{t}^{T}\left|Y_{s}-Y_{s}^{\prime}\right|^{2} d s\right)^{1 / 2}\left(\mathbb{E} \int_{t}^{T}\left|f_{s}\right|^{2} d s\right)^{1 / 2} \\
& \leq \mathbb{E}\left|\phi\left(X_{T}\right)-\phi^{\prime}\left(X_{T}\right)\right|^{2}+C\left(\sup _{t \in[0, T]} \mathbb{E}\left(1+\left|X_{t}\right|^{2 p}\right)\right)^{1 / 2}\left(\mathbb{E} \int_{t}^{T}\left|Y_{s}-Y_{s}^{\prime}\right|^{2} d s\right)^{1 / 2} .
\end{aligned}
$$

(3.5) follows immediately.

We are now able to state and prove the main result of this section, where for the process $X$ we take the Ornstein-Uhlenbeck process introduced in section 2.2: Given $x_{0} \in H$ we define

$$
X_{t}=e^{t A} x_{0}+\int_{0}^{t} e^{(t-s) A} G d W_{s}
$$

Theorem 6 Assume that Hypotheses 1 and 4 hold and suppose that the operators $e^{t B}$ are compact for $t>0$. Let $X$ be the Ornstein-Uhlenbeck process defined by (3.11).

Then there exists a mild solution $(Y, Z)$ to equation (3.1).

Moreover there exist Borel measurable functions $u:[0, T] \times H \rightarrow K, v:[0, T] \times H \rightarrow L_{2}(\Xi, K)$ such that, $\mathbb{P}$-a.s.,

$$
Y_{t}=u\left(t, X_{t}\right), \text { for all } t \in[0, T] ; \quad Z_{t}=v\left(t, X_{t}\right), \text { for almost all } t \in[0, T] .
$$


Proof - First Step. Approximation. We apply Lemma 3 to the metric space $[0, T] \times H$ and the Hilbert space $K \times L_{2}(\Xi, K)$ and obtain a sequence of functions $\psi^{n}:[0, T] \times H \times K \times$ $L_{2}(\Xi, K) \rightarrow K$ such that, for any $n \geq 1$,

$$
\left|\psi^{n}(t, x, y, z)\right| \leq C\left(1+|x|^{p}+|y|+|z|\right),
$$

and for fixed $n, \psi^{n}$ is Lipschitz with respect to $(y, z)$ uniformly with respect to $(t, x)$.

Let $\left(Y^{n, t, x}, Z^{n, t, x}\right)$ be the unique mild solution of

$$
d Y_{s}^{n, t, x}=-B Y_{s}^{n, t, x} d s-\psi^{n}\left(s, X_{s}^{t, x}, Y_{s}^{n, t, x}, Z_{s}^{n, t, x}\right) d s+Z_{s}^{n, t, x} d W_{s}, \quad Y_{T}^{n, t, x}=\phi\left(X_{T}^{t, x}\right),
$$

where $X_{s}^{t, x}$ is the Ornstein-Uhlenbeck process starting from $x$ at time $t$ :

$$
X_{s}^{t, x}=e^{(s-t) A} x+\int_{t}^{s} e^{(s-r) A} G d W_{r}, \quad 0 \leq t \leq s \leq T,
$$

(we define $X_{s}^{t, x}=x$ for $s<t$ ). It is easy to prove that $\sup _{s \in[0, T]} \mathbb{E}\left|X_{s}^{t, x}\right|^{2 p} \leq C\left(1+|x|^{2 p}\right.$ ) and (3.4) implies

$$
\sup _{s \in[t, T]} \mathbb{E}\left|Y_{s}^{n, t, x}\right|^{2}+\mathbb{E} \int_{0}^{T}\left|Z_{s}^{n, t, x}\right|^{2} d s \leq C\left(1+|x|^{2 p}\right) .
$$

Moreover there exist Borel measurable functions $u^{n}:[0, T] \times H \rightarrow K$ and $v^{n}:[0, T] \times H \rightarrow$ $L_{2}(\Xi, K)$, such that

$$
Y_{s}^{n, t, x}=u^{n}\left(s, X_{s}^{t, x}\right), \quad Z_{s}^{n, t, x}=v^{n}\left(s, X_{s}^{t, x}\right) .
$$

The proof of (3.15) can be found in [8] (see also [9], Proposition 3.2, for a direct proof in the infinite dimensional case).

Second Step. In this step we prove that there exists a subsequence of $u^{n}(t, x)$ which is convergent in $K$ for every $t, x$. This is obvious for $t=T$, since $u^{n}(T, x)=\phi(x)$, so we can assume $t<T$.

We denote by $\mu_{t}(x, d y)$ the gaussian measure $\mathcal{N}\left(e^{t A} x, Q_{t}\right)(d y)$ and by $\mu_{T}(d y)$ the measure $\mathcal{N}\left(0, Q_{T}\right)(d y)$, and we note that the law of $X_{s}^{t, x}$ is $\mu_{s-t}(x, d y), 0 \leq t \leq s \leq T$. Noting that $u^{n}(t, x)=Y_{t}^{n, t, x}$, taking expectation in the BSDE we have

$$
\begin{aligned}
u^{n}(t & , x)=\mathbb{E} e^{(T-t) B} \phi\left(X_{T}^{t, x}\right)+\mathbb{E} \int_{t}^{T} e^{(s-t) B} \psi^{n}\left(s, X_{s}^{t, x}, Y_{s}^{n, t, x}, Z_{s}^{n, t, x}\right) d s \\
= & \mathbb{E} e^{(T-t) B} \phi\left(X_{T}^{t, x}\right)+\mathbb{E} \int_{t}^{T} e^{(s-t) B} \psi^{n}\left(s, X_{s}^{t, x}, u^{n}\left(s, X_{s}^{t, x}\right), v^{n}\left(s, X_{s}^{t, x}\right)\right) d s \\
= & \mathbb{E} e^{(T-t) B} \phi\left(X_{T}^{t, x}\right)+\int_{t}^{T} e^{(s-t) B} \int_{H} \Psi^{n}(s, y) \mu_{s-t}(x, d y) d s,
\end{aligned}
$$

where $\Psi^{n}(s, y)=\psi^{n}\left(s, y, u^{n}(s, y), v^{n}(s, y)\right)$. For $t<T$ and $\delta>0$ so small that $t+\delta \leq T$ we decompose $u^{n}(t, x)$ as follows:

$$
u^{n}(t, x)=q(t, x)+a_{\delta}^{n}(t, x)+b_{\delta}^{n}(t, x),
$$

where $q(t, x)=\mathbb{E} e^{(T-t) B} \phi\left(X_{T}^{t, x}\right)$,

$$
\begin{aligned}
a_{\delta}^{n}(t, x) & =\int_{t}^{t+\delta} e^{(s-t) B} \int_{H} \Psi^{n}(s, y) \mu_{s-t}(x, d y) d s, \\
b_{\delta}^{n}(t, x) & =\int_{t+\delta}^{T} e^{(s-t) B} \int_{H} \Psi^{n}(s, y) \mu_{s-t}(x, d y) d s .
\end{aligned}
$$


We note that the inequality

$$
\begin{gathered}
\left|\int_{H} \Psi^{n}(s, y) \mu_{s-t}(x, d y)\right|=\left|\mathbb{E} \psi^{n}\left(s, X_{s}^{t, x}, Y_{s}^{n, t, x}, Z_{s}^{n, t, x}\right)\right| \\
\leq C \mathbb{E}\left(1+\left|X_{s}^{t, x}\right|^{p}+\left|Y_{s}^{n, t, x}\right|+\left|Z_{s}^{n, t, x}\right|\right)
\end{gathered}
$$

implies

$$
\begin{aligned}
\left|a_{\delta}^{n}(t, x)\right| & \leq C \mathbb{E} \int_{t}^{t+\delta}\left(1+\left|X_{s}^{t, x}\right|^{p}+\left|Y_{s}^{n, t, x}\right|+\left|Z_{s}^{n, t, x}\right|\right) d s \\
& \leq C \delta^{1 / 2}\left(\mathbb{E} \int_{t}^{t+\delta}\left(1+\left|X_{s}^{t, x}\right|^{2 p}+\left|Y_{s}^{n, t, x}\right|^{2}+\left|Z_{s}^{n, t, x}\right|^{2}\right) d s\right)^{1 / 2} \\
& \leq C_{x} \delta^{1 / 2}
\end{aligned}
$$

by (3.14). Next we consider $b_{\delta}^{n}(t, x)$ that we rewrite

$$
b_{\delta}^{n}(t, x)=\int_{t+\delta}^{T} e^{(s-t) B} \int_{H} \Psi^{n}(s, y) d^{s, t}(x, y) \mu_{s}(0, d y) d s,
$$

where we have denoted $d^{s, t}(x, y)$ the density of $\mu_{s-t}(x, \cdot)$ with respect to $\mu_{s}(0, \cdot)$. Let us consider the Hilbert space of Borel measurable functions $[0, T] \times H \rightarrow K$, square summable with respect to the measure $\mu_{s}(0, d y) d s$, equipped with the usual inner product. It will be denoted $L^{2}([0, T] \times$ $\left.H ; \mu_{s}(0, d y) d s ; K\right)$. Let us check that $\left(\Psi^{n}\right)$ is a bounded set in this space: Indeed we have

$$
\begin{aligned}
& \int_{0}^{T} \int_{H}\left|\Psi^{n}(s, y)\right|^{2} \mu_{s}(0, d y) d s=\mathbb{E} \int_{0}^{T}\left|\psi^{n}\left(s, X_{s}^{0,0}, Y_{s}^{n, 0,0}, Z_{s}^{n, 0,0}\right)\right|^{2} d s \\
& \quad \leq C \mathbb{E} \int_{0}^{T}\left(1+\left|X_{s}^{0,0}\right|^{2 p}+\left|Y_{s}^{n, 0,0}\right|^{2}+\left|Z_{s}^{n, 0,0}\right|^{2}\right) d s \\
& \quad \leq C,
\end{aligned}
$$

by (3.14). The sequence $\left(\Psi^{n}\right)$ is therefore weakly compact and there exists a subsequence (still denoted $\left.\left(\Psi^{n}\right)\right)$ which is weakly convergent in $L^{2}\left([0, T] \times H ; \mu_{s}(0, d y) d s ; K\right)$.

For fixed $k \in K$ define

$$
\varphi(s, y)=1_{[t+\delta, T]}(s) d^{s, t}(x, y) e^{(s-t) B^{*}} k
$$

and assume for a moment that $\varphi$ (which of course depends also on $t, x, \delta, k)$ belongs to $L^{2}([0, T] \times$ $\left.H ; \mu_{s}(0, d y) d s ; K\right)$. The function $\varphi$ is chosen so that

$$
\left\langle b_{\delta}^{n}(t, x), k\right\rangle=\int_{t+\delta}^{T} \int_{H}\left\langle e^{(s-t) B} \Psi^{n}(s, y), k\right\rangle d^{s, t}(x, y) \mu_{s}(0, d y) d s=\left\langle\Psi^{n}, \varphi\right\rangle_{L^{2}\left([0, T] \times H ; \mu_{s}(0, d y) d s ; K\right)} .
$$

It follows that for integers $n, m \geq 1$,

$$
\begin{gathered}
\left\langle u^{n}(t, x)-u^{m}(t, x), k\right\rangle=\left\langle a_{\delta}^{n}(t, x)-a_{\delta}^{m}(t, x), k\right\rangle+\left\langle b_{\delta}^{n}(t, x)-b_{\delta}^{m}(t, x), k\right\rangle \\
=\left\langle a_{\delta}^{n}(t, x)-a_{\delta}^{m}(t, x), k\right\rangle+\left\langle\Psi^{n}-\Psi^{m}, \varphi\right\rangle_{L^{2}\left([0, T] \times H ; \mu_{s}(0, d y) d s ; K\right)} .
\end{gathered}
$$

From (3.19) it follows that

$$
\left|\left\langle u^{n}(t, x)-u^{m}(t, x), k\right\rangle\right| \leq C \delta^{1 / 2}|k|+\left|\left\langle\Psi^{n}-\Psi^{m}, \varphi\right\rangle_{L^{2}\left([0, T] \times H ; \mu_{s}(0, d y) d s ; K\right)}\right|,
$$

and since $\left(\Psi^{n}\right)$ is weakly convergent we conclude that $\left(\left\langle u^{n}(t, x), k\right\rangle\right)_{n}$ is a Cauchy sequence for every $k \in K$, so that, for all $t, x,\left(u^{n}(t, x)\right)_{n}$ is a weakly convergent sequence in $K$. 
It remains to check that $\varphi \in L^{2}\left([0, T] \times H ; \mu_{s}(0, d y) d s ; K\right)$. From Lemma 2, the density $d^{s, t}(x, y)$ has the form

$$
\begin{gathered}
d^{s, t}(x, y)=\operatorname{det}\left(1-\Theta^{s, t}\right)^{-\frac{1}{2}} \exp \left\{-\frac{1}{2}\left\langle\left(1-\Theta^{s, t}\right)^{-1} Q_{s}^{-\frac{1}{2}} e^{(s-t) A} x, Q_{s}^{-\frac{1}{2}} e^{(s-t) A} x\right\rangle\right. \\
\left.+\left\langle\left(1-\Theta^{s, t}\right)^{-1} Q_{s}^{-\frac{1}{2}} e^{(s-t) A} x, Q_{s}^{-\frac{1}{2}} y\right\rangle-\frac{1}{2}\left\langle\Theta^{s, t}\left(1-\Theta^{s, t}\right)^{-1} Q_{s}^{-\frac{1}{2}} y, Q_{s}^{-\frac{1}{2}} y\right\rangle\right\}
\end{gathered}
$$

where $\Theta^{s, t}=Q_{s}^{-1 / 2} e^{(s-t) A} Q_{t}\left(Q_{s}^{-1 / 2} e^{(s-t) A}\right)^{*}$. So setting $h_{s, t, x}=\left(1-\Theta^{s, t}\right)^{-1} Q_{s}^{-\frac{1}{2}} e^{(s-t) A} x$, we obtain $0 \leq d^{s, t}(x, y) \leq \operatorname{det}\left(1-\Theta^{s, t}\right)^{-1 / 2} \exp \left(\left\langle h_{s, t, x}, Q_{s}^{-\frac{1}{2}} y\right\rangle\right)$ and recalling formula (2.8) we find

$$
\begin{gathered}
\int_{0}^{T} \int_{H}|\varphi(s, y)|^{2} \mu_{s}(0, d y) d s \leq C \int_{t+\delta}^{T} \int_{H}\left|d^{s, t}(x, y)\right|^{2} \mu_{s}(0, d y) d s \\
\leq C \int_{t+\delta}^{T} \operatorname{det}\left(1-\Theta^{s, t}\right)^{-1} \exp \left(2\left|h_{s, t, x}\right|^{2}\right) d s
\end{gathered}
$$

By (2.6) we have

$$
\operatorname{det}\left(1-\Theta^{s, t}\right)^{-1} \leq \exp \left[\left(1+\left|Q_{t}\right|\left|Q_{s-t}^{-\frac{1}{2}} e^{(s-t) A}\right|^{2}\right)\left|Q_{s-t}^{-\frac{1}{2}} e^{(s-t) A}\right|^{2} \operatorname{Trace} Q_{t}\right]
$$

and, taking into account (2.5),

$$
\begin{aligned}
& \left|h_{s, t, x}\right| \leq\left|\left(1-\Theta^{s, t}\right)^{-1}\right|\left|Q_{s}^{-\frac{1}{2}} Q_{s-t}^{\frac{1}{2}}\right|\left|Q_{s-t}^{-\frac{1}{2}} e^{(s-t) A}\right||x| \\
& \quad \leq\left(1+\left|Q_{t}\right|\left|Q_{s-t}^{-\frac{1}{2}} e^{(s-t) A}\right|^{2}\right)\left|Q_{s}^{-\frac{1}{2}} Q_{s-t}^{\frac{1}{2}}\right|\left|Q_{s-t}^{-\frac{1}{2}} e^{(s-t) A}\right||x| .
\end{aligned}
$$

Since $Q_{s} \geq Q_{s-t}$ it follows that $\left|Q_{s}^{-\frac{1}{2}} Q_{s-t}^{\frac{1}{2}}\right| \leq 1$. Using the inequality (7.6) and noting that $s-t \geq \delta$ we obtain $\left|Q_{s-t}^{-\frac{1}{2}} e^{(s-t) A}\right| \leq\left|Q_{\delta}^{-\frac{1}{2}} e^{\delta A}\right|$. It follows that

$$
\begin{gathered}
\operatorname{det}\left(1-\Theta^{s, t}\right)^{-1} \leq \exp \left[\left(1+\left|Q_{t}\right|\left|Q_{\delta}^{-\frac{1}{2}} e^{\delta A}\right|^{2}\right)\left|Q_{\delta}^{-\frac{1}{2}} e^{\delta A}\right|^{2} \text { Trace } Q_{t}\right], \\
\left|h_{s, t, x}\right| \leq\left(1+\left|Q_{t}\right|\left|Q_{\delta}^{-\frac{1}{2}} e^{\delta A}\right|^{2}\right)\left|Q_{\delta}^{-\frac{1}{2}} e^{\delta A}\right||x| .
\end{gathered}
$$

This shows that the right-hand side of $(3.20)$ is finite and therefore $\varphi$ belongs to $L^{2}([0, T] \times$ $\left.H ; \mu_{s}(0, d y) d s ; K\right)$.

So far in Step 2 we have proved that for all $t, x$, the sequence $\left(u^{n}(t, x)\right)_{n}$ is weakly convergent in $K$. We will now prove that the convergence takes place in the norm of $K$. To this purpose it is enough to show that, for fixed $t, x$, the sequence $\left(u^{n}(t, x)\right)_{n}$ is relatively compact in $K$ or, equivalently, that it is totally bounded.

Let us fix $(t, x)$ and let $\epsilon>0$ be arbitrary. Let us consider again the decomposition (3.17). By (3.19) we can choose $\delta$ such that $\left|a_{\delta}^{n}(t, x)\right|<\epsilon / 2$ for every $n$. Next note that

$$
b_{\delta}^{n}(t, x)=e^{\delta B} \int_{t+\delta}^{T} e^{(s-t-\delta) B} \int_{H} \Psi^{n}(s, y) \mu_{s-t}(x, d y) d s,
$$

and from (3.18) it follows that

$\left|\int_{t+\delta}^{T} e^{(s-t-\delta) B} \int_{H} \Psi^{n}(s, y) \mu_{s-t}(x, d y) d s\right| \leq C \mathbb{E} \int_{0}^{T}\left(1+\left|X_{s}^{t, x}\right|^{p}+\left|Y_{s}^{n, t, x}\right|+\left|Z_{s}^{n, t, x}\right|\right) d s \leq C(t, x, \delta)$ 
by (3.14). Since $e^{\delta B}$ is compact by our assumptions, the sequence $\left(b_{\delta}^{n}(t, x)\right)_{n}$ is relatively compact, hence totally bounded. So there exists a finite set $A \subset K$ such that for any $n$ there exists $a \in A$ satisfying $\left|b_{\delta}^{n}(t, x)-a\right|<\epsilon / 2$. So for every $n$ there exists $a \in A$ such that $\left|u^{n}(t, x)-q(t, x)-a\right|<\epsilon$. This proves that $\left(u^{n}(t, x)\right)_{n}$ is totally bounded. We have now proved that $\left(u^{n}(t, x)\right)_{n}$ is a convergent sequence in $K$ for every $(t, x)$.

Third Step. Convergence of $Y^{n}$ and $Z^{n}$.

Let us consider again the the Ornstein-Uhlenbeck process $X_{s}=X_{s}^{0, x_{0}}$ defined in (3.11) and let us denote $Y_{s}^{n}=Y_{s}^{n, 0, x_{0}}, Z_{s}^{n}=Z_{s}^{n, 0, x_{0}}$. Denoting by $u(t, x)$ the limit of $u^{n}(t, x)$ then obviously $Y_{s}^{n}=u^{n}\left(s, X_{s}\right)$ converges to $u\left(s, X_{s}\right)$, which we denote by $Y_{s}$. Setting $s=t$ in (3.14) we have $\left|u^{n}(t, x)\right|=\mathbb{E}\left|Y_{t}^{n, t, x}\right| \leq C\left(1+|x|^{p}\right)$ and consequently

$$
\left|Y_{s}^{n}\right|^{2}=\left|u^{n}\left(s, X_{s}\right)\right|^{2} \leq C\left(1+\left|X_{s}\right|^{2 p}\right)
$$

since $\mathbb{E} \int_{0}^{T}\left|X_{t}\right|^{2 p} d t<\infty$ we conclude that $Y^{n}$ converges to $Y$ in $L^{2}(\Omega \times[0, T] ; K)$. ¿From inequality (3.5) of Lemma 5 it follows that

$$
\begin{aligned}
& \mathbb{E} \int_{0}^{T}\left|Z_{t}^{n}-Z_{t}^{m}\right|^{2} d t \leq C\left(\sup _{t \in[0, T]} \mathbb{E}\left(1+\left|X_{t}\right|^{2 p}\right)\right)^{1 / 2}\left(\mathbb{E} \int_{0}^{T}\left|Y_{t}^{n}-Y_{t}^{m}\right|^{2} d t\right)^{1 / 2} \\
& \quad \leq C_{x_{0}}\left(\mathbb{E} \int_{0}^{T}\left|Y_{t}^{n}-Y_{t}^{m}\right|^{2} d t\right)^{1 / 2}
\end{aligned}
$$

from which we conclude that $\left(Z^{n}\right)$ is a Cauchy sequence in $L^{2}\left(\Omega \times[0, T] ; L_{2}(\Xi, K)\right)$. Let us denote by $Z$ its limit. Passing to a subsequence, we can assume that $\left|Z_{t}^{n}-Z_{t}\right| \rightarrow 0, \mathbb{P}$-a.s. for almost every $t$. Let us define a function $v:[0, T] \times H \rightarrow L_{2}(\Xi, K)$ by setting $v(t, x)=\lim _{n \rightarrow \infty} v^{n}(t, x)$ for all $(t, x)$ for which the limit exists, $v(t, x)=0$ elsewhere. Then $v$ is Borel measurable and we have $Z_{t}=v\left(t, X_{t}\right), \mathbb{P}$-a.s. for almost every $t$.

Fourth Step. Existence of solution. For every $t \in[0, T],\left(Y^{n}, Z^{n}\right)$ satisfies $\mathbb{P}$-a.s.:

$$
Y_{t}^{n}+\int_{t}^{T} e^{(t-s) B} Z_{s}^{n} d W_{s}=e^{(T-t) B} \phi\left(X_{T}\right)+\int_{t}^{T} e^{(t-s) B} \psi^{n}\left(s, X_{s}, Y_{s}^{n}, Z_{s}^{n}\right) d s .
$$

To prove that $(Y, Z)$ is a solution to $(3.3)$ it remains to check that

$$
\mathbb{E} \int_{0}^{T}\left|\psi^{n}\left(s, X_{s}, Y_{s}^{n}, Z_{s}^{n}\right)-\psi\left(s, X_{s}, Y_{s}, Z_{s}\right)\right| d s \rightarrow 0
$$

From (iv) of Lemma 3 we obtain $\psi^{n}\left(s, x, y_{n}, z_{n}\right) \rightarrow \psi(s, x, y, z)$ in $K$, whenever $y_{n} \rightarrow y$ in $K$ and $z_{n} \rightarrow z$ in $L_{2}(\Xi, K)$, for every $s \in[0, T], x \in H$. Taking into account (3.12) and (3.14) we have

$$
\mathbb{E} \int_{0}^{T}\left|\psi^{n}\left(s, X_{s}, Y_{s}^{n}, Z_{s}^{n}\right)\right|^{2} d s \leq C \mathbb{E} \int_{0}^{T}\left(1+\left|X_{s}\right|^{2 p}+\left|Y_{s}^{n}\right|^{2}+\left|Z_{s}^{n}\right|^{2}\right) d s \leq C
$$

which shows that $\left(\psi^{n}\left(s, X_{s}, Y_{s}^{n}, Z_{s}^{n}\right)\right)$ is uniformly integrable on $\Omega \times[0, T]$ and the required convergence follows immediately.

\section{BSDE with bounded continuous generator}

In this section and in the following one we adopt a more general approach and we consider a process $X$ with values in a metric space. We will assume that $X$ is a Markov process with respect to a Brownian filtration. More precisely, in the sequel we will make the following assumptions. 
(1) $(\Omega, \mathcal{F}, \mathbb{P})$ is a complete probability space and $\left\{W_{t}, t \in[0, T]\right\}$ is a cylindrical Wiener process in a Hilbert space $\Xi$. For an arbitrary interval $[s, t] \subset[0, T]$ we denote by $\mathcal{F}_{[s, t]}$ the $\sigma$ algebra generated by the random variables $\left\{W_{r}^{\xi}-W_{s}^{\xi}, r \in[s, t], \xi \in \Xi\right\}$ and by the $\mathbb{P}$-null sets of $\mathcal{F}$.

(2) $X=\left\{X_{s}^{t, x}(\omega), \omega \in \Omega, 0 \leq t \leq s \leq T, x \in M\right\}$ is a stochastic process with values in a complete separable metric space $M$, measurable with respect to $\mathcal{F} \times \mathcal{B}(\Delta) \times \mathcal{B}(M)$ and $\mathcal{B}(M)$ respectively (here by $\Delta$ we denote the set $\{(t, s), 0 \leq t \leq s \leq T\}$ and by $\mathcal{B}(\Lambda)$ the Borel $\sigma$-algebra of any topological space $\Lambda$ ).

(3) For every $t \in[0, T]$ and $x \in M$, the process $\left\{X_{s}^{t, x}, s \in[t, T]\right\}$ has continuous paths and is adapted to the filtration $\left\{\mathcal{F}_{[t, s]}, s \in[t, T]\right\}$.

(4) For $0 \leq t \leq s \leq T$ and $x \in M$ we have, $\mathbb{P}$-a.s.,

$$
X_{t}^{t, x}=x, \quad X_{r}^{s, X_{s}^{t, x}}=X_{r}^{t, x}, \quad \tau \in[s, T] .
$$

Let us denote by

$$
\mu_{s}^{t, x}(A)=\mathbb{P}\left(X_{s}^{t, x} \in A\right), \quad 0 \leq t \leq s \leq T, x \in M, A \in \mathcal{B}(M),
$$

the transition probabilities. Standard arguments show that $X$ is a Markov process, in the sense that for every bounded Borel function $\phi$ on $M$ and for $0 \leq t \leq s \leq r \leq T$ and $x \in M$, we have

$$
\mathbb{E}^{\mathcal{F}_{s}} \phi\left(X_{r}^{t, x}\right)=\int_{M} \phi(y) \mu_{r}^{s, X_{s}^{t, x}}(d y), \quad \mathbb{P}-\text { a.s. }
$$

We need the following lemma, that has been proved in [9], Proposition 3.2, in the special case when $M$ is a Hilbert space. Exactly the same arguments carry over to the general case.

Lemma 7 Assume the properties (1) - (4) above. Suppose that

(i) $z=\{z(\omega, s, t, x), \omega \in \Omega, 0 \leq t \leq s \leq T, x \in M\}$ is a stochastic process with values in a Hilbert space $V$, measurable with respect to $\mathcal{F} \times \mathcal{B}(\Delta) \times \mathcal{B}(M)$ and $\mathcal{B}(V)$ respectively.

(ii) For every $t \in[0, T]$ and $x \in M$, the process $\{z(s, t, x), s \in[t, T]\}$ is predictable with respect to the filtration $\left\{\mathcal{F}_{[t, s]}, s \in[t, T]\right\}$.

(iii) For $0 \leq t \leq s \leq T$ and $x \in M$ we have, $\mathbb{P}$-a.s.,

$$
z\left(r, s, X_{s}^{t, x}\right)=z(r, t, x), \quad \text { for almost all } r \in[s, T] .
$$

Then there exists a Borel measurable function $v:[0, T] \times M \rightarrow V$ such that, for $t \in[0, T]$ and $x \in H$, we have $\mathbb{P}$-a.s.

$$
z(s, t, x)=v\left(s, X_{s}^{t, x}\right), \quad \text { for almost all } s \in[t, T] .
$$

We fix arbitrary $x \in M$ and consider the following BSDE:

$$
d Y_{t}=-B Y_{t} d t-\psi\left(t, X_{t}^{0, x}, Y_{t}, Z_{t}\right) d t+Z_{t} d W_{t}, \quad Y_{T}=\phi\left(X_{T}^{0, x}\right),
$$

under the following assumptions. 
Hypothesis 8 (i) The process $X$ satisfies the properties (1)-(4) above.

(ii) The operator $B: D(B) \subset K \rightarrow K$ is the infinitesimal generator of a strongly continuous dissipative semigroup $\left\{e^{t B}, t \geq 0\right\}$ of bounded linear operators in $K$.

(iii) $\phi: M \rightarrow K$ and $\psi:[0, T] \times M \times K \times L_{2}(\Xi, K) \rightarrow K$ are Borel measurable functions,

$$
\mathbb{E}\left|\phi\left(X_{T}^{t, x}\right)\right|^{2}<\infty, \quad t \in[0, T], x \in M,
$$

and there exists a constant $C>0$ such that

$$
|\psi(t, x, y, z)| \leq C, \quad t \in[0, T], x \in M, y \in K, z \in L_{2}(\Xi, K) .
$$

(iv) For every $t \in[0, T]$ and $x \in M$ the function $\psi(t, x, \cdot, \cdot): K \times L_{2}(\Xi, K) \rightarrow K$ is continuous.

We say that an $\left(\mathcal{F}_{t}\right)$-predictable process $(Y, Z)$ with values in $K \times L_{2}(\Xi, K)$ is a mild solution of (4.4) if

$$
\sup _{t \in[0, T]} \mathbb{E}\left|Y_{t}\right|^{2}+\mathbb{E} \int_{0}^{T}\left|Z_{t}\right|^{2} d t<\infty
$$

and for every $t \in[0, T]$ the following equality holds:

$$
Y_{t}+\int_{t}^{T} e^{(t-s) B} Z_{s} d W_{s}=e^{(T-t) B} \phi\left(X_{T}^{0, x}\right)+\int_{t}^{T} e^{(t-s) B} \psi\left(s, X_{s}^{0, x}, Y_{s}, Z_{s}\right) d s, \quad \mathbb{P}-\text { a.s. }
$$

Lemma 9 Assume that Hypothesis 8 holds and let $(Y, Z)$ be a mild solution to (4.4). Then

$$
\sup _{t \in[0, T]} \mathbb{E}\left|Y_{t}\right|^{2}+\mathbb{E} \int_{0}^{T}\left|Z_{t}\right|^{2} d t \leq C\left(1+\mathbb{E}\left|\phi\left(X_{T}^{0, x}\right)\right|^{2}\right)
$$

If $\psi^{\prime}, \phi^{\prime}$ are functions satisfying Hypothesis 8 and $\left(Y^{\prime}, Z^{\prime}\right)$ is a corresponding mild solution then

$$
\mathbb{E} \int_{0}^{T}\left|Z_{t}-Z_{t}^{\prime}\right|^{2} d t \leq \mathbb{E}\left|\phi\left(X_{T}^{0, x}\right)-\phi^{\prime}\left(X_{T}^{0, x}\right)\right|^{2}+C \mathbb{E} \int_{0}^{T}\left|Y_{t}-Y_{t}^{\prime}\right| d t .
$$

In (4.7) and (4.8) the constant $C$ depends only on $T$ and on the constant $C$ in Hypothesis 8.

Proof. Proceeding as in the proof of Lemma 5 we obtain (compare (3.10))

$$
\mathbb{E}\left|Y_{t}\right|^{2}+\mathbb{E} \int_{t}^{T}\left|Z_{s}\right|^{2} d s \leq \mathbb{E}\left|\phi\left(X_{T}^{0, x}\right)\right|^{2}+2 \mathbb{E} \int_{t}^{T}\left\langle Y_{s}, \psi\left(s, X_{s}^{0, x}, Y_{s}, Z_{s}\right)\right\rangle d s .
$$

Since $\psi$ is bounded we have

$$
\begin{aligned}
\mathbb{E}\left|Y_{t}\right|^{2}+\mathbb{E} \int_{t}^{T}\left|Z_{s}\right|^{2} d s & \leq \mathbb{E}\left|\phi\left(X_{T}^{0, x}\right)\right|^{2}+C \mathbb{E} \int_{t}^{T}\left|Y_{s}\right| d s \\
& \leq \mathbb{E}\left|\phi\left(X_{T}^{0, x}\right)\right|^{2}+C \mathbb{E} \int_{t}^{T}\left(1+\left|Y_{s}\right|^{2}\right) d s
\end{aligned}
$$

and (4.7) follows from Gronwall's lemma.

In order to prove (4.8) we write the equation satisfied by $\left(Y-Y^{\prime}, Z-Z^{\prime}\right)$ and proceeding as before we arrive at

$$
\begin{aligned}
\mathbb{E} \mid Y_{t} & -\left.Y_{t}^{\prime}\right|^{2}+\mathbb{E} \int_{t}^{T}\left|Z_{s}-Z_{s}^{\prime}\right|^{2} d s \\
& \leq \mathbb{E}\left|\phi\left(X_{T}^{0, x}\right)-\phi^{\prime}\left(X_{T}^{0, x}\right)\right|^{2}+2 \mathbb{E} \int_{t}^{T}\left\langle Y_{s}-Y_{s}^{\prime}, \psi\left(s, X_{s}^{0, x}, Y_{s}, Z_{s}\right)-\psi^{\prime}\left(s, X_{s}^{0, x}, Y_{s}^{\prime}, Z_{s}^{\prime}\right)\right\rangle d s .
\end{aligned}
$$


By the boundedness assumptions on $\psi, \psi^{\prime}$ we obtain

$$
\mathbb{E}\left|Y_{t}-Y_{t}^{\prime}\right|^{2}+\mathbb{E} \int_{t}^{T}\left|Z_{s}-Z_{s}^{\prime}\right|^{2} d s \leq \mathbb{E}\left|\phi\left(X_{T}^{0, x}\right)-\phi^{\prime}\left(X_{T}^{0, x}\right)\right|^{2}+C \mathbb{E} \int_{t}^{T}\left|Y_{s}-Y_{s}^{\prime}\right| d s .
$$

(4.8) follows immediately.

Theorem 10 Assume that Hypothesis 8 holds, that the operators $e^{t B}$ are compact for $t>0$, and that the transition probabilities of the process $X$ :

$$
\mu_{s}^{t, x}, \quad 0 \leq t<s \leq T, x \in M
$$

are all equivalent measures on $M$.

Then there exists a mild solution to equation (4.4).

Moreover there exist Borel measurable functions $u:[0, T] \times M \rightarrow K, v:[0, T] \times M \rightarrow$ $L_{2}(\Xi, K)$ such that, $\mathbb{P}$-a.s.,

$$
Y_{t}=u\left(t, X_{t}\right), \text { for all } t \in[0, T] ; \quad Z_{t}=v\left(t, X_{t}\right), \text { for almost all } t \in[0, T] .
$$

Proof - First Step. Approximation. We apply Lemma 3 to the metric space $[0, T] \times M$ and the Hilbert space $K \times L_{2}(\Xi, K)$ and obtain a sequence of functions $\psi^{n}:[0, T] \times M \times K \times$ $L_{2}(\Xi, K) \rightarrow K$ such that, for any $n \geq 1$,

$$
\left|\psi^{n}(t, x, y, z)\right| \leq C,
$$

and for fixed $n, \psi^{n}$ is Lipschitz with respect to $(y, z)$ uniformly with respect to $(t, x)$.

Let $\left(Y^{n, t, x}, Z^{n, t, x}\right)$ be the unique mild solution of

$$
d Y_{s}^{n, t, x}=-B Y_{s}^{n, t, x} d s-\psi^{n}\left(s, X_{s}^{t, x}, Y_{s}^{n, t, x}, Z_{s}^{n, t, x}\right) d s+Z_{s}^{n, t, x} d W_{s}, \quad Y_{T}^{n, t, x}=\phi\left(X_{T}^{t, x}\right),
$$

where we use the convention $X_{s}^{t, x}=x$ for $s<t$. By (4.7)

$$
\sup _{s \in[t, T]} \mathbb{E}\left|Y_{s}^{n, t, x}\right|^{2}+\mathbb{E} \int_{0}^{T}\left|Z_{s}^{n, t, x}\right|^{2} d s \leq C\left(1+\mathbb{E}\left|\phi\left(X_{T}^{t, x}\right)\right|^{2}\right)<\infty .
$$

Moreover, from the uniqueness of the solution to (4.11) it is easy to deduce the following identities: for $0 \leq t \leq s \leq T$ and $x \in M$, we have, $\mathbb{P}$-a.s.,

$$
\begin{array}{ll}
Y_{r}^{n, s, X_{s}^{t, x}}=Y_{r}^{n, t, x}, & \text { for all } r \in[s, T], \\
Z_{r}^{n, s, X_{s}^{t, x}}=Z_{r}^{n, t, x}, & \text { for almost all } r \in[s, T] .
\end{array}
$$

Setting $u^{n}(t, x)=Y_{t}^{n, t, x}$ it follows immediately that for every $t, x, \mathbb{P}$-a.s.,

$$
Y_{s}^{n, t, x}=u^{n}\left(s, X_{s}^{t, x}\right), \quad s \in[t, T] .
$$

Applying Lemma 7 to the process $z(s, t, x)=Z_{s}^{n, t, x}$ we conclude that there exist Borel measurable functions $v^{n}:[0, T] \times M \rightarrow L_{2}(\Xi, K)$, such that for every $t, x, \mathbb{P}$-a.s.,

$$
Z_{s}^{n, t, x}=v^{n}\left(s, X_{s}^{t, x}\right), \quad \text { for almost all } s \in[t, T] .
$$

Second Step. In this step we prove that there exists a subsequence of $u^{n}(t, x)$ which is convergent in $K$ for every $t, x$. This is obvious for $t=T$, since $u^{n}(T, x)=\phi(x)$, so we can assume $t<T$. 
Noting that $u^{n}(t, x)=Y_{t}^{n, t, x}$, taking expectation in the BSDE we have

$$
\begin{aligned}
u^{n}(t & , x)=\mathbb{E} e^{(T-t) B} \phi\left(X_{T}^{t, x}\right)+\mathbb{E} \int_{t}^{T} e^{(s-t) B} \psi^{n}\left(s, X_{s}^{t, x}, Y_{s}^{n, t, x}, Z_{s}^{n, t, x}\right) d s \\
= & \mathbb{E} e^{(T-t) B} \phi\left(X_{T}^{t, x}\right)+\mathbb{E} \int_{t}^{T} e^{(s-t) B} \psi^{n}\left(s, X_{s}^{t, x}, u^{n}\left(s, X_{s}^{t, x}\right), v^{n}\left(s, X_{s}^{t, x}\right)\right) d s \\
= & \mathbb{E} e^{(T-t) B} \phi\left(X_{T}^{t, x}\right)+\int_{t}^{T} e^{(s-t) B} \int_{M} \Psi^{n}(s, y) \mu_{s}^{t, x}(d y) d s,
\end{aligned}
$$

where $\Psi^{n}(s, y)=\psi^{n}\left(s, y, u^{n}(s, y), v^{n}(s, y)\right)$. We fix an arbitrary $x_{0} \in M$ and note that, from our assumptions, $\mu_{s}^{t, x}$ is absolutely continuous with respect to $\mu_{s}^{0, x_{0}}$ for $s>t$ and $x \in M$. Let us denote by $d^{s, t}(x, y)$ the corresponding density. Then

$$
u^{n}(t, x)=\mathbb{E} e^{(T-t) B} \phi\left(X_{T}^{t, x}\right)+\int_{t}^{T} \int_{M} e^{(s-t) B} \Psi^{n}(s, y) d^{s, t}(x, y) \mu_{s}^{0, x_{0}}(d y) d s .
$$

Since $\left(\Psi^{n}\right)$ is uniformly bounded, this family is a bounded set in $L^{\infty}\left([0, T] \times M ; \mu_{s}^{0, x_{0}}(d y) d s ; K\right)$, whence relatively compact in the weak ${ }^{*}$ topology. Since, in addition, the space $L^{1}([0, T] \times$ $\left.M ; \mu_{s}^{0, x_{0}}(d y) d s ; K\right)$ is separable, there exists a sequence (still denoted $\Psi^{n}$ ) and a function $\Psi^{0} \in$ $L^{\infty}\left([0, T] \times M ; \mu_{s}^{0, x_{0}}(d y) d s ; K\right)$ such that for any $\varphi \in L^{1}\left([0, T] \times M ; \mu_{s}^{0, x_{0}}(d y) d s ; K\right)$ we have

$$
\lim _{n \rightarrow \infty} \int_{0}^{T} \int_{M}\left\langle\Psi^{n}(s, y)-\Psi^{0}(s, y), \varphi(s, y)\right\rangle_{K} \mu_{s}^{0, x_{0}}(d y) d s=0 .
$$

For any fixed $(t, x)$ and for every $k \in K$,

$$
\begin{aligned}
& \int_{0}^{T} \int_{M} 1_{s \in[t, T]} d^{s, t}(x, y)\left|e^{(s-t) B^{*}} k\right| \mu_{s}^{0, x_{0}}(d y) d s=\int_{t}^{T} \int_{M}\left|e^{(s-t) B^{*}} k\right| \mu_{s}^{t, x}(d y) d s \\
& \quad \leq C \int_{t}^{T} \int_{M} \mu_{s}^{t, x}(d y) d s=C \cdot(T-t),
\end{aligned}
$$

which shows that $\varphi(s, y)=1_{s \in[t, T]} d^{s, t}(x, y) e^{(s-t) B^{*}} k$ belongs to $L^{1}\left([0, T] \times M ; \mu_{s}^{0, x_{0}}(d y) d s ; K\right)$. We conclude that

$$
\begin{gathered}
\lim _{n \rightarrow \infty}\left\langle u^{n}(t, x), k\right\rangle=\mathbb{E}\left\langle e^{(s-t) B} \phi\left(X_{T}^{t, x}\right), k\right\rangle+\lim _{n \rightarrow \infty} \int_{0}^{T} \int_{M}\left\langle\Psi^{n}(s, y), \varphi(s, y)\right\rangle_{K} \mu_{s}^{0, x_{0}}(d y) d s \\
=\mathbb{E}\left\langle e^{(s-t) B} \phi\left(X_{T}^{t, x}\right), k\right\rangle+\int_{t}^{T} \int_{M}\left\langle\Psi^{0}(s, y), e^{(s-t) B^{*}} k\right\rangle d^{s, t}(x, y) \mu_{s}^{0, x_{0}}(d y) d s .
\end{gathered}
$$

and so that $\left(u^{n}(t, x)\right)_{n}$ is weakly convergent in $K$ for every $t, x$.

To prove that $\left(u^{n}(t, x)\right)_{n}$ is convergent in the norm of $K$ we will show that, for every $(t, x)$, the sequence $\left(u^{n}(t, x)\right)_{n}$ is totally bounded.

For $t<T$ and $\delta>0$ so small that $t+\delta \leq T$ we decompose $u^{n}(t, x)$ as follows (compare $(4.13))$ :

$$
u^{n}(t, x)=q(t, x)+a_{\delta}^{n}(t, x)+b_{\delta}^{n}(t, x),
$$

where $q(t, x)=\mathbb{E} e^{(T-t) B} \phi\left(X_{T}^{t, x}\right)$,

$a_{\delta}^{n}(t, x)=\int_{t}^{t+\delta} e^{(s-t) B} \int_{M} \Psi^{n}(s, y) \mu_{s}^{t, x}(d y) d s, \quad b_{\delta}^{n}(t, x)=\int_{t+\delta}^{T} e^{(s-t) B} \int_{M} \Psi^{n}(s, y) \mu_{s}^{t, x}(d y) d s$. 
Let us fix $(t, x)$ and let $\epsilon>0$ be arbitrary. Since $\left(\Psi^{n}\right)$ is uniformly bounded, we have $\left|\int_{M} \Psi^{n}(s, y) \mu_{s}^{t, x}(d y) d s\right| \leq C$, so it follows that $\left|a_{\delta}^{n}(t, x)\right| \leq C \delta$, and we can choose $\delta$ such that $\left|a_{\delta}^{n}(t, x)\right|<\epsilon / 2$ for every $n$. Next note that

$$
b_{\delta}^{n}(t, x)=e^{\delta B} \int_{t+\delta}^{T} e^{(s-t-\delta) B} \int_{M} \Psi^{n}(s, y) \mu_{s}^{t, x}(d y) d s,
$$

and

$$
\left|\int_{t+\delta}^{T} e^{(s-t-\delta) B} \int_{M} \Psi^{n}(s, y) \mu_{s}^{t, x}(d y) d s\right| \leq C .
$$

Since $e^{\delta B}$ is compact by our assumptions, the sequence $\left(b_{\delta}^{n}(t, x)\right)_{n}$ is relatively compact, hence totally bounded. So there exists a finite set $A \subset K$ such that for any $n$ there exists $a \in A$ satisfying $\left|b_{\delta}^{n}(t, x)-a\right|<\epsilon / 2$. So for every $n$ there exists $a \in A$ such that $\left|u^{n}(t, x)-q(t, x)-a\right|<\epsilon$. This shows that $\left(u^{n}(t, x)\right)_{n}$ is totally bounded and the claim is proved.

Third Step. Convergence of $Y^{n}$ and $Z^{n}$.

Let us denote $Y_{s}^{n}=Y_{s}^{n, 0, x_{0}}, Z_{s}^{n}=Z_{s}^{n, 0, x_{0}}$. Denoting by $u^{0}(t, x)$ the limit of $u^{n}(t, x)$ then obviously $Y_{s}^{n}=u^{n}\left(s, X_{s}\right)$ converges to $u\left(s, X_{s}\right)$, which we denote by $Y_{s}$. ¿From (4.12) it follows that

$$
\sup _{n} \mathbb{E} \int_{0}^{T}\left|Y_{s}^{n}\right|^{2} d s<\infty
$$

and we deduce that $Y^{n}$ converges to $Y$ in $L^{1}(\Omega \times[0, T] ; K)$. ¿From inequality (4.8) of Lemma 9 it follows that

$$
\mathbb{E} \int_{0}^{T}\left|Z_{t}^{n}-Z_{t}^{m}\right|^{2} d t \leq C \mathbb{E} \int_{0}^{T}\left|Y_{t}^{n}-Y_{t}^{m}\right| d t,
$$

from which we conclude that $\left(Z^{n}\right)$ is a Cauchy sequence in $L^{2}\left(\Omega \times[0, T] ; L_{2}(\Xi, K)\right)$. Let us denote by $Z$ its limit. Passing to a subsequence, we can assume that $\left|Z_{t}^{n}-Z_{t}\right| \rightarrow 0$, P-a.s. for almost every $t$. Let us define a function $v:[0, T] \times H \rightarrow L_{2}(\Xi, K)$ setting $v(t, x)=\lim _{n \rightarrow \infty} v^{n}(t, x)$ for all $(t, x)$ for which the limit exists, $v(t, x)=0$ elsewhere. Then $v$ is Borel measurable and we have $Z_{t}=v\left(t, X_{t}\right), \mathbb{P}$-a.s. for almost every $t$.

Fourth Step. Existence of solution. For every $t \in[0, T],\left(Y^{n}, Z^{n}\right)$ satisfies $\mathbb{P}$-a.s.:

$$
Y_{t}^{n}+\int_{t}^{T} e^{(t-s) B} Z_{s}^{n} d W_{s}=e^{(T-t) B} \phi\left(X_{T}^{0, x_{0}}\right)+\int_{t}^{T} e^{(t-s) B} \psi^{n}\left(s, X_{s}^{0, x_{0}}, Y_{s}^{n}, Z_{s}^{n}\right) d s .
$$

To prove that $(Y, Z)$ is a solution to $(3.3)$ it remains to check that

$$
\mathbb{E} \int_{0}^{T}\left|\psi^{n}\left(s, X_{s}^{0, x_{0}}, Y_{s}^{n}, Z_{s}^{n}\right)-\psi\left(s, X_{s}^{0, x_{0}}, Y_{s}, Z_{s}\right)\right| d s \rightarrow 0
$$

From (iv) of Lemma 3 we obtain $\psi^{n}\left(s, x, y_{n}, z_{n}\right) \rightarrow \psi(s, x, y, z)$ in $K$, whenever $y_{n} \rightarrow y$ in $K$ and $z_{n} \rightarrow z$ in $L_{2}(\Xi, K)$, for every $s \in[0, T], x \in H$. Taking into account (4.10) the required convergence follows from the dominated convergence theorem.

\subsection{Example}

Let $W$ be a cylindrical Wiener process in a Hilbert space $\Xi$ with Brownian filtration $\left(\mathcal{F}_{t}\right)$. Consider the following equation on the time interval $[0, T]$ for an unknown process $X$ with values in a Hilbert space $H$ :

$$
d X_{t}=A X_{t} d t+F\left(t, X_{t}\right) d t+G d W_{t}, \quad X_{0}=x,
$$


where $x \in H$, the operators $A$ and $G$ satisfy Hypothesis $1, F:[0, T] \times H \rightarrow H$ is a Borel measurable mapping such that, for some constant $C \geq 0$,

$$
\left|F(t, x)-F\left(t, x^{\prime}\right)\right| \leq C\left|x-x^{\prime}\right|, \quad|F(t, x)| \leq C(1+|x|), \quad t \in[0, T], x, x^{\prime} \in H,
$$

and there exists $\alpha>0$ such that

$$
\text { Trace } \int_{0}^{T} s^{-\alpha} e^{s A} G G^{*} e^{s A^{*}} d s<\infty
$$

(this is a stronger assumption than Hypothesis 1-(iii)).

It is well known (see e.g. [6]) that under these conditions there exists a unique mild solution, i.e. an $\left(\mathcal{F}_{t}\right)$-adapted process $X$, with continuous paths in $H$, such that, $\mathbb{P}$-a.s.,

$$
X_{t}=e^{t A} x+\int_{0}^{t} e^{(t-s) A} F\left(s, X_{s}\right) d s+\int_{0}^{t} e^{(t-s) A} G d W_{s}, \quad t \geq 0 .
$$

$X$ is unique up to indistinguishability. Let us denote by $\mu_{t}^{0, x}$ the law of $X_{t}$.

We assume further that the image of $F$ is contained in the image of $G$ and there exists $C \geq 0$ such that

$$
\left|G^{-1} F(t, x)\right| \leq C, \quad t \in[0, T], x \in H,
$$

where $G^{-1}$ denotes the pseudo-inverse of $G$. We consider the Ornstein-Uhlenbeck process $X^{\prime}$ solution of

$$
d X_{t}^{\prime}=A X_{t}^{\prime} d t+G d W_{t}, \quad X_{0}^{\prime}=x .
$$

By the Girsanov theorem, setting

$$
\rho=\exp \left(\int_{0}^{T}\left\langle G^{-1} F\left(s, X_{s}^{\prime}\right), d W_{s}\right\rangle-\frac{1}{2} \int_{0}^{T}\left|G^{-1} F\left(s, X_{s}^{\prime}\right)\right|^{2} d s\right),
$$

we have $\mathbb{E} \rho=1$ and the process $W_{t}^{\prime}=W_{t}-\int_{0}^{t} G^{-1} F\left(s, X_{s}^{\prime}\right) d s, t \in[0, T]$, is a cylindrical Wiener process with respect to the probability $\mathbb{P}^{\prime}$ admitting density $\rho$ with respect to $\mathbb{P}$. Then we have

$$
d X_{t}^{\prime}=A X_{t}^{\prime} d t+F\left(t, X_{t}^{\prime}\right) d t+G d W_{t}^{\prime}, \quad X_{0}=x
$$

and it follows that the law of $X^{\prime}$ under $\mathbb{P}^{\prime}$ is the same as the law of $X$ under $\mathbb{P}$. Since $\mathbb{P}$ and $\mathbb{P}^{\prime}$ are equivalent measures, it follows in particular that the $\mu_{t}^{0, x}$ is equivalent to $\mathcal{N}\left(e^{t A} x, Q_{t}\right)$, and therefore that $\left\{\mu_{t}^{0, x}, t \in(0, T], x \in H\right\}$ is a family of equivalent measures. In the same way one proves that the process $X_{s}^{t, x}$, solution in the mild sense to the equation

$$
d X_{s}^{t, x}=A X_{s}^{t, x} d s+F\left(s, X_{s}^{t, x}\right) d t+G d W_{s}, \quad X_{t}=x,
$$

on the interval $[t, T] \subset[0, T]$, satisfies all the requirements of Theorem 10. So if $B, \psi, \phi$ satisfy the assumptions in Hypothesis 8 and the operators $e^{t B}$ are compact for $t>0$, then there exists a mild solution to equation (4.4).

\section{BSDE with bounded continuous generator: second case}

In this section we still consider a Markov process $X=\left\{X_{s}^{t, x}, 0 \leq t \leq s \leq T, x \in M\right\}$, with values in a complete separable metric space $M$, satisfying the properties $(\mathbf{1})-(\mathbf{4})$ of section 4 . We denote by $\mu_{s}^{t, x}$ the transition probabilities of $X$. We suppose that Hypothesis 8 holds and, in addition, that the function $\phi$ is bounded. In particular the conclusions of Lemma 9 still hold. 
We fix arbitrary $x \in M$ and we consider the same BSDE as in formula (4.4)

$$
d Y_{t}=-B Y_{t} d t-\psi\left(t, X_{t}^{0, x}, Y_{t}, Z_{t}\right) d t+Z_{t} d W_{t}, \quad Y_{T}=\phi\left(X_{T}^{0, x}\right) .
$$

As before an $\left(\mathcal{F}_{t}\right)$-predictable process $(Y, Z)$ with values in $K \times L_{2}(\Xi, K)$ is called a mild solution of (5.1) if it satisfies (4.5) and (4.6).

In this section we replace the requirement of mutual absolute continuity of the transition probabilities of $X$ with a continuity assumption of the map $x \rightarrow \mu_{s}^{t, x}$ with respect to the variation norm.

More precisely we assume that for every sequence $x_{n}$ converging to $x$ in $M$ and for $0 \leq t<$ $s \leq T$ we have

$$
\operatorname{Var}\left(\mu_{s}^{t, x}-\mu_{s}^{t, x_{n}}\right) \rightarrow 0
$$

for $n \rightarrow \infty$, where $\operatorname{Var}$ denotes the total variation.

Theorem 11 Assume that Hypothesis 8 holds, that the operators $e^{t B}$ are compact for $t>0$, that the transition probabilities of the process $X$ satisfy (5.2), and that $|\phi(x)| \leq C$ for some constant $C>0$ and every $x \in M$.

Then there exists a mild solution to equation (5.1).

Moreover there exist Borel measurable functions $u:[0, T] \times M \rightarrow K, v:[0, T] \times M \rightarrow$ $L_{2}(\Xi, K)$ such that, $\mathbb{P}$-a.s.,

$$
Y_{t}=u\left(t, X_{t}\right), \text { for all } t \in[0, T] ; \quad Z_{t}=v\left(t, X_{t}\right), \text { for almost all } t \in[0, T] .
$$

Proof - First Step. Approximation. Applying Lemma 3 we construct a sequence of functions $\psi^{n}:[0, T] \times M \times K \times L_{2}(\Xi, K) \rightarrow K$ such that, for any $n \geq 1$,

$$
\left|\psi^{n}(t, x, y, z)\right| \leq C
$$

and for fixed $n, \psi^{n}$ is Lipschitz with respect to $(y, z)$ uniformly with respect to $(t, x)$. Let $\left(Y^{n, t, x}, Z^{n, t, x}\right)$ be the unique mild solution of

$$
d Y_{s}^{n, t, x}=-B Y_{s}^{n, t, x} d s-\psi^{n}\left(s, X_{s}^{t, x}, Y_{s}^{n, t, x}, Z_{s}^{n, t, x}\right) d s+Z_{s}^{n, t, x} d W_{s}, \quad Y_{T}^{n, t, x}=\phi\left(X_{T}^{t, x}\right),
$$

where we define $X_{s}^{t, x}=x$ for $s<t$. By (4.7) and the boundedness of $\phi$,

$$
\sup _{s \in[t, T]} \mathbb{E}\left|Y_{s}^{n, t, x}\right|^{2}+\mathbb{E} \int_{0}^{T}\left|Z_{s}^{n, t, x}\right|^{2} d s \leq C\left(1+\mathbb{E}\left|\phi\left(X_{T}^{t, x}\right)\right|^{2}\right) \leq C .
$$

Arguing as in the proof of Theorem 10 we deduce that there exist Borel measurable functions $u^{n}:[0, T] \times M \rightarrow K, v^{n}:[0, T] \times M \rightarrow L_{2}(\Xi, K)$, such that for every $t, x, \mathbb{P}$-a.s.,

$$
\begin{gathered}
Y_{s}^{n, t, x}=u^{n}\left(s, X_{s}^{t, x}\right), \quad s \in[t, T], \\
Z_{s}^{n, t, x}=v^{n}\left(s, X_{s}^{t, x}\right), \quad \text { for almost all } s \in[t, T] .
\end{gathered}
$$

Second Step. In this step we prove that there exists a subsequence of $u^{n}(t, x)$ which is convergent in $K$ for every $t, x$.

We first claim that for fixed $(t, x)$ there exists a subsequence $\left(n_{k}\right)$ (depending on $(t, x)$ ) such that $\left(u^{n_{k}}(t, x)\right)_{k}$ is convergent in $K$. This is obvious for $t=T$, since $u^{n}(T, x)=\phi(x)$, so we can assume $t<T$. It is enough to show that, for fixed $t, x$, the sequence $\left(u^{n}(t, x)\right)_{n}$ is relatively compact in $K$ or, equivalently, that it is totally bounded. 
From the definition of mild solution to (5.4) we obtain, taking expectation,

$$
\begin{gathered}
u^{n}(t, x)=Y_{t}^{n, t, x}=\mathbb{E} e^{(T-t) B} \phi\left(X_{T}^{t, x}\right)+\mathbb{E} \int_{t}^{T} e^{(s-t) B} \psi^{n}\left(s, X_{s}^{t, x}, Y_{s}^{n, t, x}, Z_{s}^{n, t, x}\right) d s \\
=\mathbb{E} e^{(T-t) B} \phi\left(X_{T}^{t, x}\right)+\int_{t}^{T} e^{(s-t) B} g^{n, t, x}(s) d s,
\end{gathered}
$$

where $g^{n, t, x}(s)=\mathbb{E} \psi^{n}\left(s, X_{s}^{t, x}, Y_{s}^{n, t, x}, Z_{s}^{n, t, x}\right)$ satisfies $\left|g^{n, t, x}(s)\right| \leq C$. It follows that $\left|u^{n}(t, x)\right| \leq$ $C$, i.e. the sequence $\left(u^{n}(t, x)\right)_{n}$ is uniformly bounded. For $\delta>0$ so small that $t+\delta \leq T$ we decompose $u^{n}(t, x)$ as follows:

$$
u^{n}(t, x)=q(t, x)+a_{\delta}^{n}(t, x)+b_{\delta}^{n}(t, x),
$$

where $q(t, x)=\mathbb{E} e^{(T-t) B} \phi\left(X_{T}^{t, x}\right)$,

$$
a_{\delta}^{n}(t, x)=\int_{t}^{t+\delta} e^{(s-t) B} g^{n, t, x}(s) d s, \quad b_{\delta}^{n}(t, x)=\int_{t+\delta}^{T} e^{(s-t) B} g^{n, t, x}(s) d s .
$$

Let us fix $(t, x)$ and let $\epsilon>0$ be arbitrary. We have $\left|a_{\delta}^{n}(t, x)\right| \leq C \delta$, so that we can choose $\delta$ such that $\left|a_{\delta}^{n}(t, x)\right|<\epsilon / 2$ for every $n$. Next note that

$$
b_{\delta}^{n}(t, x)=e^{\delta B} \int_{t+\delta}^{T} e^{(s-t-\delta) B} g^{n, t, x}(s) d s,
$$

and

$$
\left|\int_{t+\delta}^{T} e^{(s-t-\delta) B} g^{n, t, x}(s) d s\right| \leq C .
$$

Since $e^{\delta B}$ is compact by our assumptions, the sequence $\left(b_{\delta}^{n}(t, x)\right)_{n}$ is relatively compact, hence totally bounded. So there exists a finite set $A \subset K$ such that for any $n$ there exists $a \in A$ satisfying $\left|b_{\delta}^{n}(t, x)-a\right|<\epsilon / 2$. So for every $n$ there exists $a \in A$ such that $\left|u^{n}(t, x)-q(t, x)-a\right|<\epsilon$. This shows that $\left(u^{n}(t, x)\right)_{n}$ is totally bounded and the claim is proved.

Next note that

$$
\begin{aligned}
u^{n}(t, x) & =\mathbb{E} e^{(T-t) B} \phi\left(X_{T}^{t, x}\right)+\mathbb{E} \int_{t}^{T} e^{(s-t) B} \psi^{n}\left(s, X_{s}^{t, x}, Y_{s}^{n, t, x}, Z_{s}^{n, t, x}\right) d s \\
& =\mathbb{E} e^{(T-t) B} \phi\left(X_{T}^{t, x}\right)+\mathbb{E} \int_{t}^{T} e^{(s-t) B} \psi^{n}\left(s, X_{s}^{t, x}, u^{n}\left(s, X_{s}^{t, x}\right), v^{n}\left(s, X_{s}^{t, x}\right)\right) d s \\
& =\int_{M} e^{(T-t) B} \phi(y) \mu_{T}^{t, x}(d y)+\int_{t}^{T} e^{(s-t) B} \int_{M} \Psi^{n}(s, y) \mu_{s}^{t, x}(d y) d s,
\end{aligned}
$$

where $\Psi^{n}(s, y)=\psi^{n}\left(s, y, u^{n}(s, y), v^{n}(s, y)\right)$.

Let us fix a dense sequence $\left(t_{j}\right)$ in $[0, T]$ and a dense sequence $\left(x_{i}\right)$ in $M$. By the previous claim and a diagonal procedure we can find a subsequence $\left(n_{k}\right)$ such that $\left(u^{n_{k}}\left(t_{j}, x_{i}\right)\right)_{k}$ converges for every $i, j$. By a change of notation we can assume that the original sequence $\left(u^{n}\left(t_{j}, x_{i}\right)\right)_{n}$ is convergent for every $i, j$.

Next we fix $j$ and we prove that $\left(u^{n}\left(t_{j}, x\right)\right)_{n}$ is convergent for every $x \in M$. The assertion is trivial if $t_{j}=T$, so we assume $t_{j}<T$. We start from the inequality

$$
\begin{aligned}
\left|u^{n}\left(t_{j}, x\right)-u^{m}\left(t_{j}, x\right)\right| \leq & \left|u^{n}\left(t_{j}, x\right)-u^{n}\left(t_{j}, x_{i}\right)\right|+\left|u^{m}\left(t_{j}, x\right)-u^{m}\left(t_{j}, x_{i}\right)\right| \\
& +\left|u^{n}\left(t_{j}, x_{i}\right)-u^{m}\left(t_{j}, x_{i}\right)\right| .
\end{aligned}
$$


We have

$$
\begin{aligned}
u^{n}\left(t_{j}, x\right)-u^{n}\left(t_{j}, x_{i}\right)= & e^{(T-t) B} \int_{M} \phi(y)\left[\mu_{T}^{t_{j}, x}(d y)-\mu_{T}^{t_{j}, x_{i}}(d y)\right]+ \\
& +\int_{t_{j}}^{T} e^{(s-t) B} \int_{M} \Psi^{n}(s, y)\left[\mu_{s}^{t_{j}, x}(d y)-\mu_{s}^{t_{j}, x_{i}}(d y)\right] d s,
\end{aligned}
$$

and since $\phi$ is bounded and $\Psi^{n}$ is uniformly bounded we obtain

$$
\left|\int_{t_{j}}^{T} e^{(s-t) B} \int_{M} \Psi^{n}(s, y)\left[\mu_{s}^{t_{j}, x}(d y)-\mu_{s}^{t_{j}, x_{i}}(d y)\right] d s\right| \leq C \int_{t_{j}}^{T} \operatorname{Var}\left(\mu_{s}^{t_{j}, x}-\mu_{s}^{t_{j}, x_{i}}\right) d s,
$$

and

$$
\left|e^{(T-t) B} \int_{M} \phi(y)\left[\mu_{T}^{t_{j}, x}(d y)-\mu_{T}^{t_{j}, x_{i}}(d y)\right] d s\right| \leq C \operatorname{Var}\left(\mu_{T}^{t_{j}, x}-\mu_{T}^{t_{j}, x_{i}}\right) .
$$

We note that by (5.2) for every sequence $x_{n} \rightarrow x$ we have $\operatorname{Var}\left(\mu_{s}^{t_{j}, x}-\mu_{s}^{t_{j}, x_{n}}\right) \rightarrow 0$ for $s>t_{j}$. Since $\operatorname{Var}\left(\mu_{s}^{t_{j}, x}-\mu_{s}^{t_{j}, x_{n}}\right) \leq 2$, by the dominated convergence theorem we obtain

$$
\int_{t_{j}}^{T} \operatorname{Var}\left(\mu_{s}^{t_{j}, x}-\mu_{s}^{t_{j}, x_{n}}\right) d s \rightarrow 0 .
$$

Given $\epsilon>0$, from the previous inequalities it follows that we can choose $x_{i}$ so close to $x$ that

$$
\left|u^{n}\left(t_{j}, x\right)-u^{n}\left(t_{j}, x_{i}\right)\right| \leq \epsilon
$$

for every $n$. In a similar way one proves that $x_{i}$ can be chosen such that in addition $\mid u^{m}\left(t_{j}, x\right)-$ $u^{m}\left(t_{j}, x_{i}\right) \mid \leq \epsilon$ for every $m$, and since $\left(u^{n}\left(t_{j}, x_{i}\right)\right)_{n}$ is convergent we conclude from (5.7) that $\left(u^{n}\left(t_{j}, x\right)\right)_{n}$ is a Cauchy sequence for every $x \in M$.

Next we prove that $\left(u^{n}(t, x)\right)_{n}$ is convergent for every $t \in[0, T]$ and $x \in M$. We can assume $t<T$, otherwise the assertion is trivial. We first claim that for $t<r$ we have

$$
\left|u^{n}(t, x)-\int_{M} u^{n}(r, y) \mu_{r}^{t, x}(d y)\right| \leq C \cdot(r-t) .
$$

From (5.4) we obtain

$$
Y_{t}^{n, t, x}-Y_{r}^{n, t, x}=\int_{t}^{r} e^{(s-t) B} \psi^{n}\left(s, X_{s}^{t, x}, Y_{s}^{n, t, x}, Z_{s}^{n, t, x}\right) d s-\int_{t}^{r} e^{(s-t) B} Z_{s}^{n, t, x} d W_{s} .
$$

Taking expectation we obtain

$$
\begin{aligned}
& \mathbb{E} \int_{t}^{r} e^{(s-t) B} \psi^{n}\left(s, X_{s}^{t, x}, Y_{s}^{n, t, x}, Z_{s}^{n, t, x}\right) d s=\mathbb{E}\left[Y_{t}^{n, t, x}-Y_{r}^{n, t, x}\right] \\
& \quad=\mathbb{E}\left[u^{n}(t, x)-u^{n}\left(r, X_{r}^{t, x}\right)\right] \\
& \quad=u^{n}(t, x)-\int_{M} u^{n}(r, y) \mu_{r}^{t, x}(d y),
\end{aligned}
$$

and since $\psi^{n}$ is uniformly bounded, (5.8) follows immediately.

Then we have, for $t_{j}>t$,

$$
\begin{aligned}
\left|u^{n}(t, x)-u^{m}(t, x)\right| \leq & \left|u^{n}(t, x)-\int_{M} u^{n}\left(t_{j}, y\right) \mu_{t_{j}}^{t, x}(d y)\right| \\
& +\left|u^{m}(t, x)-\int_{M} u^{m}\left(t_{j}, y\right) \mu_{t_{j}}^{t, x}(d y)\right| \\
& +\left|\int_{M} u^{n}\left(t_{j}, y\right) \mu_{t_{j}}^{t, x}(d y)-\int_{M} u^{m}\left(t_{j}, y\right) \mu_{t_{j}}^{t, x}(d y)\right| .
\end{aligned}
$$


Given $\epsilon>0$, we choose $j$ such that $t_{j}-t<\epsilon$. For $n, m \geq N$ we have

$$
\left|u^{n}(t, x)-u^{m}(t, x)\right| \leq C \cdot \epsilon+\int_{M} \sup _{n, m \geq N}\left|u^{n}\left(t_{j}, y\right)-u^{m}\left(t_{j}, y\right)\right| \mu_{t_{j}}^{t, x}(d y) .
$$

Since the sequence $\left(u^{n}\left(t_{j}, y\right)\right)_{n}$ is convergent for every $y$ and it is uniformly bounded, the last integral tends to 0 for $N \rightarrow \infty$. The proof of step 2 is finished.

The third and fourth step are the same as in Theorem 10 and this concludes the proof.

\section{$5.1 \quad$ Example}

Let $W$ be a cylindrical Wiener process in a Hilbert space $\Xi$ with Brownian filtration $\left(\mathcal{F}_{t}\right)$. We take $H=\Xi$ and consider the following equation on the time interval $[t, T] \subset[0, T]$ for an unknown process $X$ with values in $H$ :

$$
d X_{s}=A X_{s} d s+F\left(X_{s}\right) d s+G\left(X_{s}\right) d W_{s}, \quad X_{t}=x,
$$

where $x \in H$, the operator $A: D(A) \subset H \rightarrow H$ is the infinitesimal generator of a strongly continuous semigroup $\left\{e^{t A}, t \geq 0\right\}$ of bounded linear operators in $H, F: H \rightarrow H$ and $G: H \rightarrow$ $L(H)$ are Borel measurable mappings such that, for some constant $C \geq 0$,

$$
\left|F(x)-F\left(x^{\prime}\right)\right| \leq C\left|x-x^{\prime}\right|, \quad\left|G(x)-G\left(x^{\prime}\right)\right|_{L(H)} \leq C\left|x-x^{\prime}\right|, \quad x, x^{\prime} \in H .
$$

We also assume that $e^{t A} \in L_{2}(H, H)$ for $t>0$ and that $\int_{0}^{T} e^{-\alpha t}\left|e^{t A}\right|_{L_{2}(H, H)}^{2} d t<\infty$ for some $\alpha>0$. It is well known (see e.g. [6]) that under these conditions there exists a mild solution i.e. an $\left(\mathcal{F}_{t}\right)$-adapted process, with continuous paths in $H$, such that, $\mathbb{P}$-a.s.,

$$
X_{s}=e^{(s-t) A} x+\int_{t}^{s} e^{(s-r) A} F\left(X_{r}\right) d r+\int_{t}^{s} e^{(s-r) A} G\left(X_{r}\right) d W_{r}, \quad s \in[t, T] .
$$

$X$ is unique up to indistinguishability. The solution will be denoted $X_{s}^{t, x}$, to stress the dependence on $x$ and $t$. The process $X$ constructed in this way satisfies the conditions $(\mathbf{1})-(\mathbf{4})$ of section 4 . We denote by $\mu_{s}^{t, x}$ the law of $X_{s}^{t, x}$.

Assume now in addition that $G(x)$ is invertible for every $x \in H$ and there exists $C \geq 0$ such that $\left|G(x)^{-1}\right|_{L(H)} \leq C$ for all $x \in H$. Then the following inequality has been proved in [20] (see also [7], Theorem 7.1.1 and Lemma 7.1.5):

$$
\operatorname{Var}\left(\mu_{s}^{t, x}-\mu_{s}^{t, x^{\prime}}\right) \leq \frac{C}{\sqrt{s-t}}\left|x-x^{\prime}\right|, \quad 0 \leq t<s \leq T, x, x^{\prime} \in H .
$$

So under the previous assumptions condition (5.2) clearly holds, and so if $B, \psi, \phi$ satisfy the other requirements in Theorem 11 then there exists a mild solution to equation (5.1).

\section{$6 \quad$ A stochastic game with infinitely many players}

Let $W$ be a cylindrical Wiener process in a Hilbert space $\Xi$, defined on a complete probability space $(\Omega, \mathcal{F}, \mathbb{P})$, and let $\left(\mathcal{F}_{t}\right)$ be its Brownian filtration.

We consider the Ornstein-Uhlenbeck process in a Hilbert space $H$ defined by the equation $d X_{t}=A X_{t} d t+G d W_{t}$, more precisely

$$
X_{t}=e^{t A} x+\int_{0}^{t} e^{(t-s) A} G d W_{s}, \quad t \in[0, T],
$$

with $A$ and $G$ satisfying Hypothesis 1 , and $x \in H$. 
Hypothesis $\mathbf{1 2}$ i) Let I be a finite or countable set.

ii) For every $i \in I$, a metric space $U_{i}$ is given. We denote $U=\times_{i \in I} U_{i}$ the product space.

iii) We assume that Borel measurable functions are given

$$
R:[0, T] \times H \times U \rightarrow \Xi, \quad l^{i}:[0, T] \times H \times U \rightarrow \mathbb{R}, \quad \phi^{i}: H \rightarrow \mathbb{R},
$$

for every $i \in I$. Moreover there exist constants $c_{R} \geq 0, c_{i} \geq 0$ such that

$$
|R(t, x, v)| \leq c_{R}, \quad\left|l^{i}(t, x, v)\right|+\left|\phi^{i}(x)\right| \leq c_{i}\left(1+|x|^{p}\right), \quad t \in[0, T], x \in H, v \in U, i \in I .
$$

Finally we assume that for every $t \in[0, T], x \in H$ and $i \in I$ the functions

$$
R(t, x, \cdot): U \rightarrow \Xi, \quad l^{i}(t, x, \cdot): U \rightarrow \mathbb{R}
$$

are continuous.

iv) For every $i \in I$ a number $\lambda_{i} \geq 0$ is given. If I is infinite, identifying $I$ with the natural numbers, we assume that $\lambda_{i} \rightarrow+\infty$ as $i \rightarrow \infty$.

Each element $i \in I$ represents a player. $U_{i}$ represents the set of actions that player $i$ can take at any time. Coordinates of an element $v \in U$ are denoted $v^{i}$ and we use the notation $v=\left(v^{i}\right)_{i}$.

$\lambda_{i}$ is a discount factor in the cost of player $i$, as defined below.

An $\left(\mathcal{F}_{t}\right)$-adapted process $u=\left\{u_{t}, t \in[0, T]\right\}$, with values in $U$, is called admissible decision process. Each component $u^{i}=\left\{u_{t}^{i}, t \in[0, T]\right\}, i \in I$, is then a process with values in $U_{i} ; u_{t}^{i}$ represents the action taken by player $i$ at time $t$.

For every admissible decision process $u$, a cost $J^{i}(u)$ for the player $i \in I$ is defined as follows. By the Girsanov theorem the process

$$
W_{t}^{u}=W_{t}-\int_{0}^{t} R\left(s, X_{s}, u_{s}\right) d s, \quad t \in[0, T]
$$

is a Wiener process under the probability measure $\mathbb{P}^{u}$ admitting the density $\rho^{u}$ with respect to $\mathbb{P}$ given by

$$
\rho^{u}=\exp \left(\int_{0}^{T}\left\langle R\left(s, X_{s}, u_{s}\right), d W_{s}\right\rangle-\frac{1}{2} \int_{0}^{T}\left|R\left(s, X_{s}, u_{s}\right)\right|^{2} d s\right)
$$

We define

$$
J^{i}(u)=\mathbb{E}^{u}\left[\int_{0}^{T} e^{-\lambda_{i} t} l^{i}\left(t, X_{t}, u_{t}\right) d t+e^{-\lambda_{i} T} \phi^{i}\left(X_{T}\right)\right], \quad i \in I .
$$

Since $R$ is bounded, the application of the Girsanov theorem is justified and we also have $\mathbb{E}\left|\rho^{u}\right|^{p}<\infty$ for every $p \in[1, \infty)$. We note that $X$ satisfies

$$
X_{t}=e^{t A} x+\int_{0}^{t} e^{(t-s) A} G R\left(s, X_{s}, u_{s}\right) d s+\int_{0}^{t} e^{(t-s) A} G d W_{s}^{u}, \quad t \in[0, T] .
$$

Therefore, under $\mathbb{P}^{u}, X$ is the solution of a controlled stochastic equation with nonlinear drift.

An admissible decision process $\widehat{u}$ is called a Nash equilibrium if, for each $i \in I$, the equality

$$
J^{i}(\widehat{u}) \leq J^{i}(u),
$$

takes place for arbitrary decision process $u$ satisfying, for all $j \neq i$,

$$
u_{t}^{j}=\widehat{u}_{t}^{j}, \quad \mathbb{P}-\text { a.s. for almost every } t \in[0, T] .
$$


The aim of this section is to show that a Nash equilibrium exists under appropriate conditions. Our main assumption is Hypothesis 13 below. Before its statement we introduce some notation.

Let us fix numbers $\rho_{i}>0$ such that $\sum_{i \in I} c_{i}^{2} \rho_{i}<\infty$, where $c_{i}$ are the constants introduced in Hypothesis 12. In the sequel we will consider backward equations for processes with values in the Hilbert space $\ell_{\rho}^{2}(I)$, the space of real sequences $\left(y^{i}\right)_{i}$ satisfying $\sum_{i \in I}\left|y^{i}\right|^{2} \rho_{i}<\infty$, endowed with the inner product

$$
\langle y, v\rangle_{\ell_{\rho}^{2}(I)}=\sum_{i \in I} y^{i} v^{i} \rho_{i}, \quad y=\left(y^{i}\right)_{i} \in \ell_{\rho}^{2}(I), v=\left(v^{i}\right)_{i} \in \ell_{\rho}^{2}(I) .
$$

For $i \in I$ we denote $g_{i}$ the element of $\ell_{\rho}^{2}(I)$ defined by $g_{i}^{j}=0$ if $i \neq j, g_{i}^{i}=1 / \rho_{i}$. We note that $\left\langle y, g_{i}\right\rangle_{\ell_{\rho}^{2}(I)}=y^{i}$ for every $y=\left(y^{i}\right)_{i} \in \ell_{\rho}^{2}(I)$ and that the family $\left\{g_{i} \sqrt{\rho_{i}}, i \in I\right\}$ is a complete orthonormal basis of $\ell_{\rho}^{2}(I)$. For every $z \in L_{2}\left(\Xi, \ell_{\rho}^{2}(I)\right)$ we can define elements $z^{i} \in \Xi^{*}$ by the formula

$$
z^{i} \xi=\left\langle z \xi, g_{i}\right\rangle_{\ell_{\rho}^{2}(I)}, \quad \xi \in \Xi, i \in I
$$

Since $z$ is a Hilbert-Schmidt operator we have

$$
\sum_{i \in I}\left|z^{i}\right|_{\Xi^{*}}^{2} \rho_{i}<\infty
$$

so that the sequence $\left(z^{i}\right)_{i}$ belongs to the Hilbert space $\ell_{\rho}^{2}\left(I, \Xi^{*}\right)$ consisting of $\Xi^{*}$-valued sequences satisfying (6.2), endowed with the natural inner product. It is easy to check that the mapping $z \rightarrow\left(z^{i}\right)_{i}$ is a Hilbert space isomorphism between $L_{2}\left(\Xi, \ell_{\rho}^{2}(I)\right)$ and $\ell_{\rho}^{2}\left(I, \Xi^{*}\right)$. In the sequel we will make the identification $z=\left(z^{i}\right)_{i}$.

Hypothesis 13 There exists a Borel measurable function $\underline{u}:[0, T] \times H \times L_{2}\left(\Xi, \ell_{\rho}^{2}(I)\right) \rightarrow U$ such that for every $t \in[0, T], x \in H, z=\left(z^{i}\right)_{i} \in L_{2}\left(\Xi, \ell_{\rho}^{2}(I)\right), i \in I$ the inequality

$$
z^{i} R(t, x, \underline{u}(t, x, z))+l^{i}(t, x, \underline{u}(t, x, z)) \leq z^{i} R(t, x, v)+l^{i}(t, x, v),
$$

holds for every $v \in U$ satisfying $v^{j}=\underline{u}^{j}(t, x, z)$ for all $j \neq i$. Moreover for every $t \in[0, T]$, $x \in H$ and $i \in I$ the function $\underline{u}^{i}(t, x, \cdot): L_{2}\left(\Xi, \ell_{\rho}^{2}(I)\right) \rightarrow U$ is continuous.

Remark 14 Hypotheses 12 and 13 are easier to check in the special case

$$
R(t, x, v)=\sum_{j \in I} R_{j}\left(t, x, v^{j}\right), \quad l^{i}(t, x, v)=\sum_{j \in I} l_{j}^{i}\left(t, x, v^{j}\right), \quad t \in[0, T], x \in H, v \in U,
$$

i.e. when $R$ and each $l^{i}$ are sums of functions depending only on one coordinate $v^{j} \in U_{j}$ of $v \in U$. More precisely suppose that $I, U_{i}, \phi^{i}$ satisfy the assumptions of Hypothesis 12 (in particular, $\left|\phi^{i}(x)\right| \leq \widehat{c}_{i}\left(1+|x|^{p}\right)$ for every $x, i$ and for some constants $\left.\widehat{c}_{i} \geq 0\right)$ and that for every $i, j \in I$ there exist Borel measurable functions

$$
R_{j}:[0, T] \times H \times U_{j} \rightarrow \Xi, \quad l_{j}^{i}:[0, T] \times H \times U_{j} \rightarrow \mathbb{R},
$$

and constants $c_{R j}, c_{i j}$ such that

$$
\left|R_{j}(t, x, a)\right| \leq c_{R j}, \quad\left|l_{j}^{i}(t, x, a)\right| \leq c_{i j}\left(1+|x|^{p}\right), \quad t \in[0, T], x \in H, a \in U_{j},
$$

and $\sum_{j} c_{R j}<\infty, \sum_{j} c_{i j}<\infty$ for every $i \in I$. We also assume that for every $t \in[0, T], x \in H$ and $i, j \in I$ the functions

$$
R_{j}(t, x, \cdot): U_{j} \rightarrow \Xi, \quad l_{j}^{i}(t, x, \cdot): U_{j} \rightarrow \mathbb{R}
$$


are continuous. If $R$ and $l^{i}$ are defined by (6.3) then Hypothesis 12 is satisfied with $c_{i}=$ $\sum_{j} c_{i j}+\widehat{c}_{i}$. Suppose now that there exist Borel measurable functions $\underline{u}^{i}:[0, T] \times H \times \Xi^{*} \rightarrow U_{i}$, $i \in I$, such that

$$
\eta R_{i}\left(t, x, \underline{u}^{i}(t, x, \eta)\right)+l_{i}^{i}\left(t, x, \underline{u}^{i}(t, x, \eta)\right) \leq \eta R_{i}(t, x, a)+l_{i}^{i}(t, x, a),
$$

for every $i \in I, t \in[0, T], x \in H, \eta \in \Xi^{*}, a \in U_{i}$. Moreover assume that for every $t \in[0, T]$, $x \in H$ and $i \in I$ the function $\underline{u}^{i}(t, x, \cdot): \Xi^{*} \rightarrow U_{i}$ is continuous. Then setting

$$
\underline{u}(t, x, z)=\left(\underline{u}^{i}\left(t, x, z^{i}\right)\right)_{i}
$$

it is easy to verify that Hypothesis 13 is satisfied.

Note that (6.4) can be expressed as

$$
\underline{u}^{i}(t, x, \eta) \in \underset{a \in U_{i}}{\operatorname{argmin}}\left[\eta R_{i}(t, x, a)+l_{i}^{i}(t, x, a)\right] .
$$

The existence of a function $\underline{u}^{i}$ satisfying (6.4) and such that $\underline{u}^{i}(t, x, \cdot)$ is continuous can be effectively checked in particular cases. For instance, in addition to the previous assumptions, suppose that all the metric spaces $U_{i}$ coincide with the ball $B(0, r)$ of radius $r>0$ centered at the origin of another Hilbert space $\mathcal{A}$. Furthermore assume that $R_{j}$ are defined by

$$
R_{j}(t, x, a)=\bar{R}_{j}(t, x) a, \quad t \in[0, T], x \in H, a \in \mathcal{A},
$$

where each $\bar{R}_{j}(t, x)$ is a linear bounded operator from $\mathcal{A}$ to $\Xi, \bar{R}_{j}(\cdot, \cdot) a:[0, T] \times H \rightarrow \Xi$ is Borel measurable for every $a \in \mathcal{A}$, and $\left|\bar{R}_{j}(t, x)\right| \leq \bar{c}_{R j}, t \in[0, T], x \in H$, for some constants $\bar{c}_{R j} \geq 0$ satisfying $\sum_{j} \bar{c}_{R j}<\infty$. Suppose finally that $l_{i}^{i}$ have the special form $l_{i}^{i}(t, x, a)=|a|^{2}$, $a \in B(0, r)$. Then a minimizer of $a \rightarrow \eta R_{i}(t, x, a)+l_{i}^{i}(t, x, a)=\eta \bar{R}_{i}(t, x) a+|a|^{2}$ over $B(0, r)$ can be easily computed, and the required function $\underline{u}^{i}$ can be defined by

$$
\underline{u}^{i}(t, x, \eta)=\left\{\begin{array}{lll}
-\frac{1}{2}\left(\eta \bar{R}_{i}(t, x)\right)^{*} & \text { if } & \left|\eta \bar{R}_{i}(t, x)\right| \leq 2 r, \\
-r \frac{\left(\eta \bar{R}_{i}(t, x)\right)^{*}}{\left|\eta \bar{R}_{i}(t, x)\right|} & \text { if } & \left|\eta \bar{R}_{i}(t, x)\right|>2 r
\end{array}\right.
$$

for $t \in[0, T], x \in H, \eta \in \Xi^{*}$, where by $\left(\eta \bar{R}_{i}(t, x)\right)^{*} \in \mathcal{A}$ we denote the image of $\eta \bar{R}_{i}(t, x) \in \mathcal{A}^{*}$ under the Riesz isometry $\mathcal{A}^{*} \rightarrow \mathcal{A}$.

Theorem 15 Under Hypotheses 1, 12 and 13 there exists a Nash equilibrium $\widehat{u}$. Moreover there exists a Borel measurable function $v:[0, T] \times H \rightarrow L_{2}\left(\Xi, \ell_{\rho}^{2}(I)\right)$ such that

$$
\widehat{u}_{t}=\underline{u}\left(t, X_{t}, v\left(t, X_{t}\right)\right), \quad \mathbb{P}-\text { a.s. for almost every } t \in[0, T] .
$$

Remark 16 By equality (6.5), $\widehat{u}$ is called a closed-loop Nash equilibrium.

Proof. Let us define an operator $B$ in $\ell_{\rho}^{2}(I)$ setting $(B y)_{i}=-\lambda_{i} y^{i}$ for $y \in D(B)=\left\{\left(y^{i}\right)_{i}\right.$ : $\left.\sum_{i \in I} \lambda_{i}^{2}\left|y^{i}\right|^{2} \rho_{i}<\infty\right\}$. $B$ is a self-adjoint operator with eigenvectors $g_{i}$ and eigenvalues $-\lambda_{i}$. It is the infinitesimal generator of the dissipative semigroup given by the formula $\left(e^{t B} y\right)_{i}=e^{-\lambda_{i} t} y^{i}$. The condition $\lambda_{i} \rightarrow \infty$ ensures that $e^{t B}$ is compact for every $t>0$.

Let us define $\phi(x)=\left(\phi^{i}(x)\right)_{i}$ and $f(t, x, z)=\left(f^{i}(t, x, z)\right)_{i}$, where

$$
f^{i}(t, x, z)=z^{i} R(t, x, \underline{u}(t, x, z))+l^{i}(t, x, \underline{u}(t, x, z)), \quad t \in[0, T], x \in H, z \in L_{2}\left(\Xi, \ell_{\rho}^{2}(I)\right),
$$


and let us consider the backward equation

$$
d Y_{t}=-B Y_{t} d t-f\left(t, X_{t}, Z_{t}\right) d t+Z_{t} d W_{t}, \quad Y_{T}=\phi\left(X_{T}\right),
$$

where the unknown processes $Y$ and $Z$ take values in $\ell_{\rho}^{2}(I)$ and $L_{2}\left(\Xi, \ell_{\rho}^{2}(I)\right)$ respectively.

Next we verify that the functions

$$
f:[0, T] \times H \times L_{2}\left(\Xi, \ell_{\rho}^{2}(I)\right) \rightarrow \ell_{\rho}^{2}(I), \quad \phi: H \rightarrow \ell_{\rho}^{2}(I)
$$

satisfy the assumptions of Theorem 6. By Hypothesis 12,

$$
\begin{aligned}
&|f(t, x, z)|_{\ell_{\rho}^{2}(I)} \leq\left(\sum_{i}\left|z^{i} R(t, x, \underline{u}(t, x, z))\right|^{2} \rho_{i}\right)^{1 / 2}+\left(\sum_{i}\left|l^{i}(t, x, \underline{u}(t, x, z))\right|^{2} \rho_{i}\right)^{1 / 2} \\
& \leq c_{R}\left(\sum_{i}\left|z^{i}\right|_{\Xi^{*}}^{2} \rho_{i}\right)^{1 / 2}+\left(\sum_{i} c_{i}^{2} \rho_{i}\right)^{1 / 2}\left(1+|x|^{p}\right) \\
&=c_{R}|z|_{L_{2}\left(\Xi, \ell_{\rho}^{2}(I)\right)}+\left(\sum_{i} c_{i}^{2} \rho_{i}\right)^{1 / 2}\left(1+|x|^{p}\right), \\
&|\phi(x)|_{\ell_{\rho}^{2}(I)}=\left(\sum_{i}\left|\phi^{i}(x)\right|^{2} \rho_{i}\right)^{1 / 2} \leq\left(\sum_{i} c_{i}^{2} \rho_{i}\right)^{1 / 2}\left(1+|x|^{p}\right) .
\end{aligned}
$$

The functions $f^{i}(t, x, \cdot)$ are continuous since they are defined in terms of the continuous mappings $R(t, x, \cdot), l^{i}(t, x, \cdot)$ and $\underline{u}(t, x, \cdot)$. To check continuity of $f(t, x, \cdot)$, let us consider a sequence $z_{n}$ converging to $z$ in $L_{2}\left(\Xi, \ell_{\rho}^{2}(I)\right)$ and note that

$$
\begin{aligned}
\left|f^{i}\left(t, x, z_{n}\right)-f^{i}(t, x, z)\right| \leq & \left|z_{n}^{i} R\left(t, x, \underline{u}\left(t, x, z_{n}\right)\right)-z^{i} R(t, x, \underline{u}(t, x, z))\right| \\
& +\left|l^{i}\left(t, x, \underline{u}\left(t, x, z_{n}\right)\right)-l^{i}(t, x, \underline{u}(t, x, z))\right| \\
\leq & c_{R}\left|z_{n}^{i}-z^{i}\right|+\left|z^{i}\right|\left|R\left(t, x, \underline{u}\left(t, x, z_{n}\right)\right)-R(t, x, \underline{u}(t, x, z))\right| \\
& +\left|l^{i}\left(t, x, \underline{u}\left(t, x, z_{n}\right)\right)-l^{i}(t, x, \underline{u}(t, x, z))\right| .
\end{aligned}
$$

It follows that

$$
\begin{aligned}
\left|f\left(t, x, z_{n}\right)-f(t, x, z)\right|_{\ell_{\rho}^{2}(I)}= & \left(\sum_{i}\left|f^{i}\left(t, x, z_{n}\right)-f^{i}(t, x, z)\right|^{2} \rho_{i}\right)^{1 / 2} \\
\leq & c_{R}\left|z_{n}-z\right|_{L_{2}\left(\Xi, \ell_{\rho}^{2}(I)\right)} \\
& +\left(\sum_{i}\left|z^{i}\right|^{2}\left|R\left(t, x, \underline{u}\left(t, x, z_{n}\right)\right)-R(t, x, \underline{u}(t, x, z))\right|^{2} \rho_{i}\right)^{1 / 2} \\
& +\left(\sum_{i}\left|l^{i}\left(t, x, \underline{u}\left(t, x, z_{n}\right)\right)-l^{i}(t, x, \underline{u}(t, x, z))\right|^{2} \rho_{i}\right)^{1 / 2} .
\end{aligned}
$$

Since $R$ is bounded, $\sum_{i}\left|z^{i}\right|^{2} \rho_{i}<\infty,\left|l^{i}\left(t, x, \underline{u}\left(t, x, z_{n}\right)\right)\right| \leq c_{i}\left(1+|x|^{p}\right)$ and $\sum_{i} c_{i}^{2} \rho_{i}<\infty$ we conclude that $\left|f\left(t, x, z_{n}\right)-f(t, x, z)\right|_{\ell_{\rho}^{2}(I)} \rightarrow 0$.

Theorem 6 shows that (6.7) has a solution satisfying, in particular, $\mathbb{E} \int_{0}^{T}\left|Z_{s}^{i}\right|^{2} d s<\infty$. Moreover, there exists a Borel measurable function $v:[0, T] \times H \rightarrow L_{2}\left(\Xi, \ell_{\rho}^{2}(I)\right)$ such that $Z_{t}=v\left(t, X_{t}\right), \mathbb{P}$-a.s. for almost every $t \in[0, T]$.

We will show that the process $\widehat{u}_{t}=\underline{u}\left(t, X_{t}, Z_{t}\right)=\underline{u}\left(t, X_{t}, v\left(t, X_{t}\right)\right), t \in[0, T]$, is a Nash equilibrium. Writing (6.7) in the form specified by definition (3.3) and taking scalar product with $g_{i}$ we obtain, for every $i \in I$,

$$
Y_{t}^{i}+\int_{t}^{T} e^{-\lambda_{i}(s-t)} Z_{s}^{i} d W_{s}=e^{-\lambda_{i}(T-t)} \phi^{i}\left(X_{T}\right)+\int_{t}^{T} e^{-\lambda_{i}(s-t)} f^{i}\left(s, X_{s}, Z_{s}\right) d s .
$$


For every admissible decision process $u$, by the definition of $W^{u}$ we obtain $Y_{0}^{i}-e^{-\lambda_{i} T} \phi^{i}\left(X_{T}\right)=-\int_{0}^{T} e^{-\lambda_{i} s} Z_{s}^{i} d W_{s}^{u}-\int_{0}^{T} e^{-\lambda_{i} s} Z_{s}^{i} R_{s}\left(s, X_{s}, u_{s}\right) d s+\int_{0}^{T} e^{-\lambda_{i} s} f^{i}\left(s, X_{s}, Z_{s}\right) d s$.

We recall that $W^{u}$ is a Wiener process under $\mathbb{P}^{u}$ and we note that

$$
\mathbb{E}^{u}\left(\int_{0}^{T}\left|Z_{s}^{i}\right|^{2} d s\right)^{1 / 2}=\mathbb{E}\left[\rho^{u}\left(\int_{0}^{T}\left|Z_{s}^{i}\right|^{2} d s\right)^{1 / 2}\right] \leq\left(\mathbb{E}\left|\rho^{u}\right|^{2}\right)^{1 / 2}\left(\mathbb{E} \int_{0}^{T}\left|Z_{s}^{i}\right|^{2} d s\right)^{1 / 2}<\infty .
$$

It follows that $\int_{0}^{t} Z_{s}^{i} d W_{s}^{u}, t \in[0, T]$ is a $\mathbb{P}^{u}$-martingale. Taking expectation we obtain

$$
\begin{aligned}
Y_{0}^{i} & =e^{-\lambda_{i} T} \mathbb{E}^{u} \phi^{i}\left(X_{T}\right)+\mathbb{E}^{u} \int_{0}^{T} e^{-\lambda_{i} s}\left[f^{i}\left(s, X_{s}, Z_{s}\right)-Z_{s}^{i} R\left(s, X_{s}, u_{s}\right)\right] d s \\
& =J^{i}(u)+\mathbb{E}^{u} \int_{0}^{T} e^{-\lambda_{i} s}\left[f^{i}\left(s, X_{s}, Z_{s}\right)-Z_{s}^{i} R\left(s, X_{s}, u_{s}\right)-l^{i}\left(s, X_{s}, u_{s}\right)\right] d s .
\end{aligned}
$$

By the definition of $f^{i}$ and Hypothesis 13, for every $i \in I$,

$$
f^{i}(t, x, z) \leq z^{i} R(t, x, v)+l^{i}(t, x, v), \quad t \in[0, T], x \in H, z \in L_{2}\left(\Xi, \ell_{\rho}^{2}(I)\right),
$$

for every $v \in U$ satisfying $v^{j}=\underline{u}^{j}(t, x, z)$ for all $j \neq i$. It follows that

$$
f^{i}\left(t, X_{t}, Z_{t}\right) \leq Z_{t}^{i} R\left(t, X_{t}, u_{t}\right)+l^{i}\left(t, X_{t}, u_{t}\right)
$$

for every decision process such that $u_{t}^{j}=\widehat{u}_{t}^{j}=\underline{u}^{j}\left(t, X_{t}, Z_{t}\right)$ for all $j \neq i$.

On the other hand from (6.6) we obtain

$$
f^{i}\left(t, X_{t}, Z_{t}\right)=Z_{t}^{i} R\left(t, X_{t}, \widehat{u}_{t}\right)+l^{i}\left(t, X_{t}, \widehat{u}_{t}\right)
$$

From (6.8) and (6.9) it follows that $Y_{0}^{i} \leq J^{i}(u)$; from (6.8) and (6.10) it follows that $Y_{0}^{i}=J^{i}(\widehat{u})$; we conclude that $J^{i}(\widehat{u}) \leq J^{i}(u)$, which shows that $\widehat{u}$ is a Nash equilibrium.

\section{Appendix.}

This appendix is devoted to the proof of Lemma 2. We follow closely [4], proof of Proposition 4.2. We keep the notation of section 2.2 ; by Im we denote the image of an operator. We first state a lemma on gaussian measures.

Lemma 17 Suppose that $Q, R$ are nonnegative, injective, trace class linear operators on $H$ satisfying

$$
\operatorname{Im} Q^{1 / 2}=\operatorname{Im} R^{1 / 2}
$$

suppose moreover that the operator

$$
G=\left(R^{-1 / 2} Q^{1 / 2}\right)^{*} R^{-1 / 2} Q^{1 / 2}-1
$$

is trace class. Then $\mathcal{N}(0, R)$ is equivalent to $\mathcal{N}(0, Q)$ and, for $\mathcal{N}(0, Q)$-a.e. $x \in H$,

$$
\frac{d \mathcal{N}(0, R)}{d \mathcal{N}(0, Q)}(x)=\operatorname{det}(1+G)^{1 / 2} \exp \left(-\frac{1}{2}\left\langle G Q^{-1 / 2} x, Q^{-1 / 2} x\right\rangle\right) .
$$


The determinant is understood as the infinite product of eigenvalues. It is well defined, since $G$ is trace class. Equivalence of measures follows from the Feldman-Hajek Theorem, while the formula for the density can be found in [5], II.4.3, Remark 4.4 and formula (4.16). A simple direct proof can be found in [4].

In the rest of this appendix we assume that Hypothesis 1 holds. We state two well-known properties of the operators $Q_{t}$, whose short proofs are reported for the reader's convenience.

(i) The operators $Q_{t}, t>0$, are injective.

Indeed, by a duality argument (see for instance [6], appendix B), Hypothesis 1-(iv) implies that for every $t>0$ there exists $C_{t}>0$ such that

$$
\left|e^{t A^{*}} y\right| \leq C_{t}\left|Q_{t}^{1 / 2} y\right|, \quad y \in H
$$

So if $Q_{t} x=0$ for some $t>0$, then $Q_{s} x=0, s \leq t$, and consequently $e^{s A^{*}} x=0, s \leq t$; letting $s \rightarrow 0$, we obtain $x=0$.

(ii) For every $t>0, \operatorname{Im} Q_{T}^{1 / 2}=\operatorname{Im} Q_{t}^{1 / 2}$. In particular, $Q_{T}^{-1 / 2} e^{t A}$ is a linear bounded operator on $H$.

We notice the equality $Q_{T}=Q_{t}+e^{t A} Q_{T-t} e^{t A^{*}}$, which is a consequence of the definition of $Q_{t}$ and $Q_{T}$. We obtain

$$
Q_{T}=Q_{t}+e^{t A} Q_{T-t} e^{t A^{*}}=Q_{t}^{1 / 2}\left[1+\left(Q_{t}^{-1 / 2} e^{t A}\right) Q_{T-t}\left(Q_{t}^{-1 / 2} e^{t A}\right)^{*}\right] Q_{t}^{1 / 2},
$$

which yields, for some constant $C_{t T}>0$,

$$
\left|Q_{T}^{1 / 2} x\right|^{2}=\left|\left[1+\left(Q_{t}^{-1 / 2} e^{t A}\right) Q_{T-t}\left(Q_{t}^{-1 / 2} e^{t A}\right)^{*}\right]^{1 / 2} Q_{t}^{1 / 2} x\right|^{2} \leq C_{t T}\left|Q_{t}^{1 / 2} x\right|^{2}, \quad x \in H .
$$

On the other hand,

$$
\left|Q_{t}^{1 / 2} x\right|^{2}=\left\langle Q_{t} x, x\right\rangle \leq\left\langle Q_{T} x, x\right\rangle=\left|Q_{T}^{1 / 2} x\right|^{2}, \quad x \in H .
$$

By a duality argument (see e.g. [6], Appendix B) we conclude that $\operatorname{Im} Q_{T}^{1 / 2}=\operatorname{Im} Q_{t}^{1 / 2}$.

(iii) For $0<s \leq t$ we have

$$
\left|Q_{t}^{-1 / 2} e^{t A}\right| \leq\left|Q_{s}^{-1 / 2} e^{s A}\right| .
$$

We start from the easily verified identity $Q_{t}=Q_{t-s}+e^{(t-s) A} Q_{s} e^{(t-s) A^{*}}$, which implies $Q_{t} \geq e^{(t-s) A} Q_{s} e^{(t-s) A^{*}}$ and therefore $\left|Q_{t}^{1 / 2} x\right|^{2} \geq\left|Q_{s}^{1 / 2} e^{(t-s) A^{*}} x\right|^{2}, x \in H$. By a duality argument it follows that $\left|Q_{t}^{-1 / 2} e^{(t-s) A} Q_{s}^{1 / 2}\right| \leq 1$ and consequently $\left|Q_{t}^{-1 / 2} e^{t A} x\right|=$ $\left|Q_{t}^{-1 / 2} e^{(t-s) A} Q_{s}^{1 / 2} Q_{s}^{-1 / 2} e^{s A} x\right| \leq\left|Q_{s}^{-1 / 2} e^{s A} x\right|$, which proves the claim.

Proof of Lemma 2. The kernel $k$ is the Radon-Nikodym density

$$
k_{t}(x, \cdot)=\frac{d \mathcal{N}\left(e^{t A} x, Q_{t}\right)}{d \mathcal{N}\left(0, Q_{T}\right)} .
$$

We will first prove the special case corresponding to $x=0$, namely that

$$
k_{t}(0, \cdot)=\operatorname{det}\left(1-\Theta_{t T}\right)^{-1 / 2} \exp \left\{-\frac{1}{2}\left\langle\Theta_{t T}\left(1-\Theta_{t T}\right)^{-1} Q_{T}^{-1 / 2} y, Q_{T}^{-1 / 2} y\right\rangle\right\} .
$$


Since $Q_{T-t}$ is a trace class operator and $Q_{T}^{-1 / 2} e^{t A}$ is linear bounded, the operator $\Theta_{t T}$ is trace class. Moreover, since

$$
Q_{t}=Q_{T}-e^{t A} Q_{T-t} e^{t A^{*}}=Q_{T}^{1 / 2}\left[1-\left(Q_{T}^{-1 / 2} e^{t A}\right) Q_{T-t}\left(Q_{T}^{-1 / 2} e^{t A}\right)^{*}\right] Q_{T}^{1 / 2}=Q_{T}^{1 / 2}\left(1-\Theta_{t T}\right) Q_{T}^{1 / 2}
$$

we have

$$
\left(1-\Theta_{t T}\right) x=Q_{T}^{-1 / 2} Q_{t} Q_{T}^{-1 / 2} x, \quad x \in \operatorname{Im} Q_{T}^{1 / 2} .
$$

Therefore, $\left\langle\left(1-\Theta_{t T}\right) x, x\right\rangle \geq 0$ for $x \in \operatorname{Im} Q_{T}^{1 / 2}$, a dense subset of $H$; it follows that $\left(1-\Theta_{t T}\right)$ is nonnegative. Equality (7.8) also implies, by standard arguments, that $\left(1-\Theta_{t T}\right)$ is invertible and

$$
\left(1-\Theta_{t T}\right)^{-1}=\left(Q_{t}^{-1 / 2} Q_{T}^{1 / 2}\right)^{*} Q_{t}^{-1 / 2} Q_{T}^{1 / 2} .
$$

Define $G=\left(Q_{t}^{-1 / 2} Q_{T}^{1 / 2}\right)^{*} Q_{t}^{-1 / 2} Q_{T}^{1 / 2}-1$. Then

$$
G=\left(1-\Theta_{t T}\right)^{-1}-1=\Theta_{t T}\left(1-\Theta_{t T}\right)^{-1},
$$

so $G$ is trace class and formula (7.7) follows from Lemma 17.

To prove the general case, we use the equality

$$
k_{t}(x, \cdot)=\frac{d \mathcal{N}\left(e^{t A} x, Q_{t}\right)}{d \mathcal{N}\left(0, Q_{t}\right)} \frac{d \mathcal{N}\left(0, Q_{t}\right)}{d \mathcal{N}\left(0, Q_{T}\right)}=\frac{d \mathcal{N}\left(e^{t A} x, Q_{t}\right)}{d \mathcal{N}\left(0, Q_{t}\right)} k_{t}(0, \cdot)
$$

and we notice that, by the Cameron-Martin Theorem (see e.g. [6]),

$$
\frac{d \mathcal{N}\left(e^{t A} x, Q_{t}\right)}{d \mathcal{N}\left(0, Q_{t}\right)}(y)=\exp \left(\left\langle Q_{t}^{-1 / 2} e^{t A} x, Q_{t}^{-1 / 2} y\right\rangle-\frac{1}{2}\left|Q_{t}^{-1 / 2} e^{t A} x\right|^{2}\right),
$$

for $\mathcal{N}\left(0, Q_{t}\right)$-a.e. $y \in H$. If $m \in \operatorname{Im} Q_{t}$, then (7.9) implies $\left(1-\Theta_{t T}\right)^{-1} Q_{T}^{-1 / 2} m=Q_{T}^{1 / 2} Q_{t}^{-1} m$ and we have, for $y \in H$, a.e. with respect to $\mathcal{N}\left(0, Q_{T}\right)$ and $\mathcal{N}\left(0, Q_{t}\right)$,

$$
\begin{aligned}
& \left\langle Q_{t}^{-1 / 2} m, Q_{t}^{-1 / 2} y\right\rangle=\left\langle Q_{t}^{-1} m, y\right\rangle=\left\langle Q_{T}^{1 / 2} Q_{t}^{-1} m, Q_{T}^{-1 / 2} y\right\rangle \\
& =\left\langle\left(1-\Theta_{t T}\right)^{-1} Q_{T}^{-1 / 2} m, Q_{T}^{-1 / 2} y\right\rangle .
\end{aligned}
$$

(7.9) also implies

$$
\left|Q_{t}^{-1 / 2} m\right|^{2}=\left|\left(1-\Theta_{t T}\right)^{-1 / 2} Q_{T}^{-1 / 2} m\right|^{2} .
$$

The equalities (7.12) and (7.13) extend by continuity to every $m \in \operatorname{Im} Q_{t}^{1 / 2}$. So we can set $m=e^{t A} x$, and substituting into (7.11) and using (7.7), we prove the formula for $k$.

It remains to prove the inequalities (2.5) and (2.6).

The equality (7.9) shows that $\left|\left(1-\Theta_{t T}\right)^{-1}\right|=\left|Q_{t}^{-1 / 2} Q_{T}^{1 / 2}\right|^{2}$. The first equality in (7.4) implies that

$$
\left|Q_{t}^{-1 / 2} Q_{T}^{1 / 2}\right|^{2} \leq\left|1+\left(Q_{t}^{-1 / 2} e^{t A}\right) Q_{T-t}\left(Q_{t}^{-1 / 2} e^{t A}\right)^{*}\right|,
$$

and since $\left(Q_{t}^{-1 / 2} e^{t A}\right) Q_{T-t}\left(Q_{t}^{-1 / 2} e^{t A}\right)^{*} \geq 0$, we conclude that

$$
\left|\left(1-\Theta_{t T}\right)^{-1}\right| \leq 1+\left|\left(Q_{t}^{-1 / 2} e^{t A}\right) Q_{T-t}\left(Q_{t}^{-1 / 2} e^{t A}\right)^{*}\right| \leq 1+\left|Q_{T-t}\right|\left|Q_{t}^{-1 / 2} e^{t A}\right|^{2},
$$

which proves (2.5).

In the sequel we denote for simplicity

$$
a=\left|Q_{T-t}\right|\left|Q_{t}^{-1 / 2} e^{t A}\right|^{2}
$$


To prove (2.6) we first recall that $\Theta_{t T}$ is a trace class nonnegative operator and we denote $\lambda_{0}, \lambda_{1}, \ldots$ its eigenvalues, arranged in decreasing order. Since $0 \leq \Theta_{t T}<1$ we have $0 \leq \ldots \leq$ $\lambda_{1} \leq \lambda_{0}=\left|\Theta_{t T}\right|<1$. It follows that $\left(1-\lambda_{0}\right)^{-1}=\left|\left(1-\Theta_{t T}\right)^{-1}\right|$ and by (7.14) we have $\left(1-\lambda_{0}\right)^{-1} \leq 1+a$ and we first conclude that $\lambda_{0} \leq a /(1+a)$.

Next we compute

$$
\operatorname{det}\left(1-\Theta_{t T}\right)^{-1}=\prod_{k=0}^{\infty}\left(1-\lambda_{k}\right)^{-1}=\exp \left[-\sum_{k=0}^{\infty} \log \left(1-\lambda_{k}\right)\right] .
$$

Since the function $x \rightarrow(-\log (1-x)) / x$ is increasing in the interval $(0,1)$ we have in particular

$$
\frac{-\log \left(1-\lambda_{k}\right)}{\lambda_{k}} \leq \frac{-\log \left(1-\lambda_{0}\right)}{\lambda_{0}} \leq \frac{-\log \left(1-\frac{a}{1+a}\right)}{\frac{a}{1+a}}=\frac{\log (1+a)}{a}(1+a) \leq 1+a,
$$

and we obtain

$$
\operatorname{det}\left(1-\Theta_{t T}\right)^{-1} \leq \exp \left[(1+a) \sum_{k=0}^{\infty} \lambda_{k}\right]=\exp \left[(1+a) \operatorname{Trace} \Theta_{t T}\right]
$$

Then we have

$$
\operatorname{Trace} \Theta_{t T}=\operatorname{Trace}\left(\left(Q_{T}^{-1 / 2} e^{t A}\right) Q_{T-t}\left(Q_{T}^{-1 / 2} e^{t A}\right)^{*}\right) \leq\left(\operatorname{Trace} Q_{T-t}\right)\left|Q_{T}^{-1 / 2} e^{t A}\right|^{2}
$$

and since the inequality (7.5) implies that $\left|Q_{T}^{-1 / 2} Q_{t}^{1 / 2}\right| \leq 1$, we deduce that

$$
\left|Q_{T}^{-1 / 2} e^{t A}\right| \leq\left|Q_{T}^{-1 / 2} Q_{t}^{-1 / 2}\right|\left|Q_{t}^{-1 / 2} e^{t A}\right| \leq\left|Q_{t}^{-1 / 2} e^{t A}\right|
$$

Substituting, we obtain $\operatorname{det}\left(1-\Theta_{t T}\right)^{-1} \leq \exp \left[(1+a)\left(\operatorname{Trace} Q_{T-t}\right)\left|Q_{t}^{-1 / 2} e^{t A}\right|^{2}\right]$ and (2.6) is proved.

Acknowledgments. The authors would like to thank the two anonymous referees for their helpful comments.

\section{References}

[1] F. Confortola, Dissipative backward stochastic differential equations in infinite dimensions, PhD Thesis, University of Milan, 2004.

[2] F. Confortola, Dissipative backward stochastic differential equations in infinite dimensions. Infin. Dimens. Anal. Quantum Probab. Relat. Top. 9 (2006), no. 1, 155-168.

[3] F. Confortola, Dissipative backward stochastic differential equation with locally Lipschitz nonlinearity. To appear in Stochastic Process. Appl.

[4] G. Da Prato, M. Fuhrman and J. Zabczyk, A note on regularizing properties of Ornstein-Uhlenbeck semigroups in infinite dimensions. In: G. Da Prato and L. Tubaro (Eds.) Stochastic Partial Differential Equations and Applications, pp. 167-182, Marcel Dekker, New York, 2002.

[5] Yu. Dalecky and S.V. Fomin, Measures and differential equations in infinitedimensional space. Kluwer Academic Publishers, 1991. 
[6] G. Da Prato And J. Zabczyk, Stochastic equations in infinite dimensions. Encyclopedia of Mathematics and its Applications, Cambridge University Press, 1992.

[7] G. Da Prato and J. Zabczyk, Ergodicity for infinite-dimensional systems. London Mathematical Society Lecture Note Series, 229. Cambridge University Press, Cambridge, 1996.

[8] N. El Karoui, S. Peng and M.C. Quenez, Backward stochastic differential equations in finance. Math. Finance 7 (1997), 1-71.

[9] M. Fuhrman, A class of stochastic optimal control problems in Hilbert spaces: BSDEs and optimal control laws, state constraints, conditioned processes. Stochastic Process. Appl. 108 (2003), 263-298.

[10] M. Fuhrman and G. Tessitore, Nonlinear Kolmogorov equations in infinite dimensional spaces: the backward stochastic differential equations approach and applications to optimal control. Ann. Probab. 30 (2002), 1397-1465.

[11] M. Fuhrman and G. Tessitore, Infinite horizon backward stochastic differential equations and elliptic equations in Hilbert spaces. Ann. Probab. 32 (2004), 607-660.

[12] M. Fuhrman and G. Tessitore, The Bismut-Elworthy formula for backward SDEs and applications to nonlinear Kolmogorov equations and control in infinite dimensional spaces. Stoch. Stoch. Rep. 74 (2002), 429-464.

[13] G. Guatteri, On a class of forward-backward stochastic differential systems in infinite dimensions. J. Appl. Math. Stoch. Anal. 2007, Art. ID 42640, 33 pp.

[14] G. Guatteri and G. Tessitore, On the backward stochastic Riccati equation in infinite dimensions. SIAM J. Control and Optim. 44 (2005) 159-194.

[15] S. Hamadene, J.P. Lepeltier and S. Peng, BSDEs with continuous coefficients and stochastic differential games. In: N. El Karoui and L. Mazliak (Eds.), Backward Stochastic Differential Equations (Paris, 1995-1996), Pitman Research Notes in Mathematics Series, Vol. 364, pp. 115-128. Longman, Harlow, 1997.

[16] Y. Hu AND J. MA, Nonlinear Feynman-Kac formula and discrete-functional-type BSDEs with continuous coefficients. Stochastic Process. Appl. 112 (2004), 23-51.

[17] Y. Hu And S. Peng, Adapted solution of a backward semilinear stochastic evolution equation. Stochastic Anal. Appl. 9 (1991), 445-459.

[18] E. Pardoux And S. Peng, Adapted solution of a backward stochastic differential equation. Systems Control Lett. 14 (1990), 61-74.

[19] E. Pardoux and A. Rascanu, Backward stochastic variational inequalities. Stochastics Stochastics Rep. 67 (1999), no. 3-4, 159-167.

[20] S. Peszat And J. Zabczyk, Strong Feller property and irreducibility for diffusions on Hilbert spaces. Ann. Probab. 23 (1995), 157-172.

[21] B. ØKsendal And T. Zhang, On backward stochastic partial differential equations. Preprint, 2001. 
[22] I. Simão, A conditioned Ornstein-Uhlenbeck process on a Hilbert space. Stochastic Anal. Appl. 9 (1991), 85-98.

[23] I. Simão, Regular fundamental solution for a parabolic equation on an infinite-dimensional space. Stochastic Anal. Appl. 11 (1993), 235-247.

[24] I. Simão, Regular transition densities for infinite-dimensional diffusions. Stochastic Anal. Appl. 11 (1993), 309-336. 